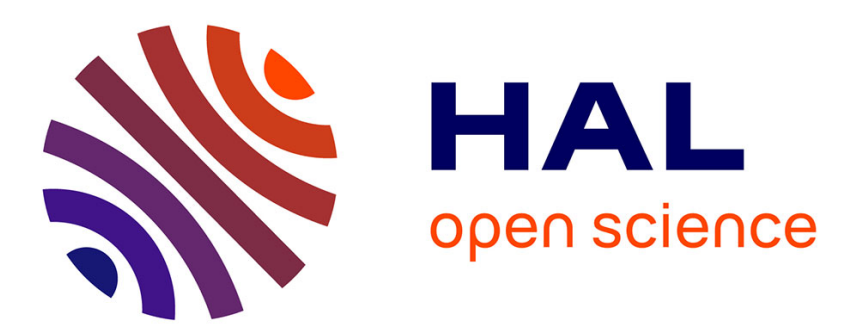

\title{
Contribution à l'étude du pompage optique par échange de métastabilité dans 3 He. - Première Partie
}

\author{
J. Dupont-Roc, M. Leduc, F. Laloë
}

\section{To cite this version:}

J. Dupont-Roc, M. Leduc, F. Laloë. Contribution à l'étude du pompage optique par échange de métastabilité dans 3He. - Première Partie. Journal de Physique, 1973, 34 (11-12), pp.961-976. 10.1051/jphys:019730034011-12096100 . jpa-00208119

HAL Id: jpa-00208119

https://hal.science/jpa-00208119

Submitted on 1 Jan 1973

HAL is a multi-disciplinary open access archive for the deposit and dissemination of scientific research documents, whether they are published or not. The documents may come from teaching and research institutions in France or abroad, or from public or private research centers.
L'archive ouverte pluridisciplinaire HAL, est destinée au dépôt et à la diffusion de documents scientifiques de niveau recherche, publiés ou non, émanant des établissements d'enseignement et de recherche français ou étrangers, des laboratoires publics ou privés. 


\title{
CONTRIBUTION A L'ÉTUDE DU POMPAGE OPTIQUE PAR ÉCHANGE DE MÉTASTABILITÉ DANS ${ }^{3} \mathrm{He}$
}

\author{
Première Partie \\ J. DUPONT-ROC, M. LEDUC et F. LALOË \\ Laboratoire de Spectroscopie Hertzienne de l'ENS \\ 24, rue Lhomond, 75005 Paris, France
}

(Reçu le 14 juin 1973)

\begin{abstract}
Résumé. - On étudie théoriquement l'effet des collisions d'échange de métastabilité dans ${ }^{3} \mathrm{He}$ sur les variables internes des atomes, dans l'état fondamental $1{ }^{1} \mathrm{~S}_{0}$ et dans l'état métastable $2{ }^{3} \mathrm{~S}_{1}$. La méthode adoptée ici consiste à utiliser directement les équations qui régissent l'évolution de l'orientation et de l'alignement dans les différents niveaux. On parvient ainsi à une description théorique détaillée et relativement simple du mécanisme de pompage optique "indirect » de ${ }^{3} \mathrm{He}$. L'étude du couplage entre les orientations longitudinales des différents niveaux permet de rendre compte de la cinétique de ce pompage, en particulier du temps de construction de l'orientation nucléaire dans le niveau fondamental. En ce qui concerne les orientations transversales, on calcule en détail les effets de " circulation de cohérence ", qui se traduisent par exemple par un déplacement des fréquences de résonance des différents niveaux.
\end{abstract}

\begin{abstract}
We investigate theoretically the effect of metastability exchange collisions on the internal variables of ${ }^{3} \mathrm{He}$ atoms in the $1{ }^{1} \mathrm{~S}_{0}$ ground state and in the $2{ }^{3} \mathrm{~S}_{1}$ metastable state. The method used here is to derive directly the equations of motion of different atomic observables (orientation, alignment). We are thus able to rather simply theoretically describe the indirect optical pumping process of ${ }^{3} \mathrm{He}$. The study of the coupling between the longitudinal orientations of the different levels involved in the process leads to a description of the kinetics of the pumping process and particularly of the building time of the ground state nuclear orientation. Regarding transverse orientations, we calculate in detail the effects of coherence circulation, which produce among other things a shift of the resonance frequency of the different atomic levels.
\end{abstract}

De nombreux éléments ont pu être orientés dans leur niveau fondamental par pompage optique direct au moyen de leur raie de résonance. Toutefois, cette méthode ne s'applique pas à ${ }^{3} \mathrm{He}$, de spin nucléaire $I=\frac{1}{2}$, car la raie de résonance de l'hélium est située dans l'ultra-violet trop lointain $(\lambda=585 \AA)$. C'est pourquoi Colegrove, Schearer et Walters [1] ont mis au point une méthode de pompage optique " indirect ", qui consiste à orienter au moyen de la raie $\lambda=10830 \AA$ le niveau métastable $2{ }^{3} \mathrm{~S}_{1}$, l'orientation ainsi obtenue étant ensuite transférée au niveau fondamental par les collisions dites d'échange de métastabilité (collisions de transfert d'excitation entre un atome d'hélium dans le niveau métastable et un autre dans le niveau fondamental). L'échange de métastabilité dans l'hélium joue donc un rôle pratique important, et on comprend l'intérêt qui s'est attaché à son étude.
D'un point de vue théorique, le potentiel $V(R)$ d'interaction entre un atome d'hélium dans le niveau $2{ }^{3} \mathrm{~S}_{1}$ et un autre dans le niveau fondamental, situés à une distance $R$, a fait l'objet de nombreuses études. Depuis les travaux de Buckingham et Dalgarno [2], qui ont en particulier prévu l'existence d'une barrière répulsive dans le potentiel, divers calculs ont été effectués [3], [4] pour améliorer la connaissance de $V(R)$. Une fois que l'on connaît ce potentiel, on peut théoriquement en déduire la section efficace d'échange de métastabilité $\sigma_{\text {ech }}$; cependant, les valeurs ainsi trouvées dépendent de façon critique des variations avec $R$ de $V(R)$, notamment à grande distance, et de la hauteur de la barrière de potentiel. Les déterminations expérimentales de $\sigma_{\text {ech }}$ présentent donc un grand intérêt, puisqu'elles fournissent un test de la validité du potentiel $V(R)$ calculé.

A l'heure actuelle, ce sont les expériences de 
pompage optique dans l'hélium qui permettent d'obtenir avec la meilleure précision la valeur de $\sigma_{\text {ech }}$, ainsi que ses variations avec la température [5]; de plus, elles permettent également de mesurer le coefficient de diffusion d'un atome métastable $2{ }^{3} \mathrm{~S}_{1}$ dans un gaz d'hélium, c'est-à-dire la section efficace de diffusion élastique $\sigma_{\mathrm{d}}$ [6], [7]. C'est pourquoi les calculs récents cherchent-ils à obtenir un potentiel $V(R)$ conduisant à la fois à des valeurs de $\sigma_{\text {ech }}$ et $\sigma_{\mathrm{d}}$ en accord avec l'expérience ([8] à [11]).

Dans cet article l'accent sera mis, non sur le calcul du potentiel d'interaction $V(R)$ et celui de la valeur de $\sigma_{\text {ech }}$, mais sur l'effet des collisions d'échange de métastabilité sur les variables internes des atomes (spin électronique et nucléaire des atomes dans les niveaux fondamental et métastable). Cette étude est nécessaire pour arriver à une compréhension détaillée $\mathrm{du}$ mécanisme du pompage optique dans ${ }^{3} \mathrm{He}$, ce qui permet par exemple de calculer des valeurs correctes de $\sigma_{\text {ech }}$ à partir des données expérimentales; comme nous l'avons déjà signalé dans une publication antérieure [12], il convient de multiplier par un facteur $\frac{9}{4}$ les valeurs de $\sigma_{\text {ech }}$ précédemment admises; ceci modifie en conséquence la comparaison avec les données expérimentales qui permet de tester la validité d'un potentiel $V(R)$ obtenu à partir de considérations théoriques. Diverses autres caractéristiques du pompage optique de ${ }^{3} \mathrm{He}$ seront étudiées dans cet article. L'étude du couplage entre les orientations longitudinales des différents niveaux nous conduira à une description précise de la cinétique du pompage optique « indirect » dans ${ }^{3} \mathrm{He}$ et notamment au calcul de la constante de temps longue $T_{1}$ de construction de l'orientation nucléaire $\left(T_{1} \simeq 100 \mathrm{~s}\right)$. En ce qui concerne les orientations transversales, nous examinerons comment l'échange de métastabilité peut conduire à des effets de "circulation de cohérence », qui sont en fait proches de certains phénomènes déjà bien connus dans le cas d'un pompage optique direct par la raie de résonance [13].

Un certain nombre d'auteurs ont étudié, théoriquement ou expérimentalement, plusieurs des problèmes qui nous intéressent ici; nous les citerons dans le texte de cet article, au fur et à mesure que ces problèmes seront abordés. Cependant, nous utiliserons ici une approche différente de la leur pour étudier l'évolution des atomes soumis au pompage optique. En effet, on décrit habituellement cette évolution en écrivant les équations d'évolution des populations des différents sous-niveaux Zeeman des niveaux hyperfins $F=\frac{3}{2}$ et $F=\frac{1}{2}$ du niveau métastable $2{ }^{3} \mathrm{~S}_{1}$, ainsi que du niveau fondamental ; ceci conduit à un système de 8 équations non linéaires couplées, c'est-à-dire à un système relativement compliqué, bien que soluble dans certains cas. Quant aux cohérences Zeeman des matrices densité, elles obéissent à des équations encore moins maniables. Or, dans une expérience de pompage optique, on ne détecte pas telle population ou telle cohérence, mais des combinaisons linéaires de ces quantités, qui sont les valeurs moyennes d'observables atomiques (orientations, alignement). La méthode que nous proposons ici est d'utiliser directement les équations qui régissent l'évolution de ces observables; bien que cette façon de faire soit en théorie équivalente à la précédente, elle apporte en pratique une grande simplification et, de plus, conduit à un point de vue plus physique. Nous verrons ainsi que l'on peut donner une description théorique complète et relativement simple du pompage optique dans ${ }^{3} \mathrm{He}$, bien que les phénomènes soient plus complexes pour un pompage indirect que dans le cas habituel.

Rappels : conditions expérimentales du pompage optique dans ${ }^{3} \mathrm{He}$ - - La figure 1 représente le schéma des premiers niveaux de l'atome ${ }^{3} \mathrm{He}$ : le niveau fondamental $1{ }^{1} \mathrm{~S}_{0}$ possède un moment cinétique d'origine purement nucléaire (le spin nucléaire $I$ vaut $\frac{1}{2}$ ); le niveau métastable $2{ }^{3} \mathrm{~S}_{1}$ se compose de deux sousniveaux hyperfins $F=\frac{3}{2}$ et $F=\frac{1}{2}$, approximativement distants de $6600 \mathrm{MHz}$. Dans une cellule scellée contenant du gaz de ${ }^{3} \mathrm{He}$ sous faible pression $(0,1 \leqslant p \leqslant 5$ torr $)$, on entretient une décharge peu intense de manière à porter un certain nombre d'atomes dans le niveau métastable (la densité d'atomes métastables est $n \simeq 10^{10}$ à $10^{11}$ atomes $/ \mathrm{cm}^{3}$, celle d'atomes fondamentaux $N \simeq 10^{15}$ à $10^{17}$ atomes $/ \mathrm{cm}^{3}$ ).
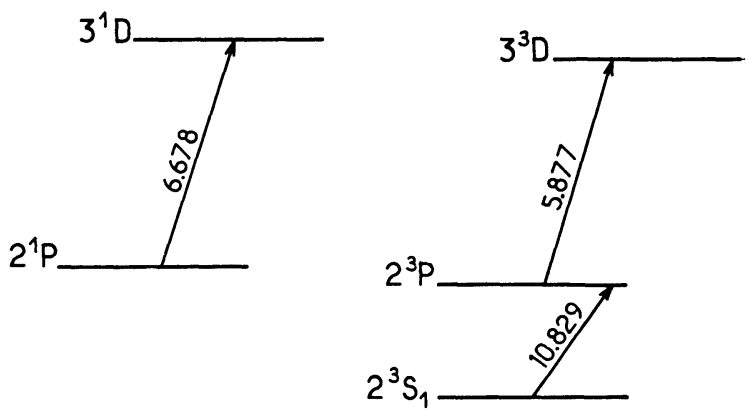

$1^{1} S_{0}$

Fig. 1. - Schéma des premiers niveaux excités de l'atome d'hélium. La figure n'est pas à l'échelle. Les longueurs d'onde sont données en angström.

Les atomes métastables sont orientés par voie optique, au moyen de la raie $\lambda=10830 \AA$ (transition $2{ }^{3} \mathrm{~S}-2{ }^{3} \mathrm{P}$, voir Fig. 1); pour cela, on éclaire la cellule à l'aide d'un faisceau lumineux polarisé circulairement issu d'une lampe à hélium. Comme nous l'avons déjà dit plus haut, ce sont les collisions d'échange de métastabilité qui assurent un fort couplage entre les orientations des niveaux métastable $2{ }^{3} \mathrm{~S}_{1}$ et fondamental $1{ }^{1} \mathrm{~S}_{0}$; il en résulte une importante polarisation nucléaire du niveau fondamental (de l'ordre de $5 \%$ ) 
Pour détecter optiquement cette orientation nucléaire, plusieurs techniques sont possibles. On utilise souvent la mesure de l'absorption par les atomes métastables d'un faisceau de longueur d'onde $\lambda=10830 \AA$ circulairement polarisé (par exemple le faisceau de pompage); les coïncidences isotopiques entre ${ }^{3} \mathrm{He}$ et ${ }^{4} \mathrm{He}$ sont telles que, lorsque ce faisceau est issu d'une lampe à ${ }^{4} \mathrm{He}$, on observe l'orientation du sous-niveau $F=\frac{3}{2}$ du niveau métastable [14], [15], le couplage dû à l'échange de métastabilité imposant à cette orientation d'être proportionnelle à celle du niveau fondamental. Une variante de cette méthode consiste à mesurer l'alignement du niveau $F=\frac{3}{2}$ [16], [17] (le faisceau de détection est alors linéairement polarisé) ; son inconvénient pour détecter l'orientation nucléaire est de donner des signaux proportionnels au carré de cette orientation (cf. $\S 1.2 .3$ ), et par suite très faibles. Dans les expériences décrites plus bas $(\S 5)$, nous utilisons une autre méthode, mise au point antérieurement [18], qui consiste à observer la polarisation circulaire de certaines raies optiques de l'hélium (par exemple $\lambda=5876 \AA$ ou $6678 \AA$, voir Fig. 1) émises par la décharge : on utilise le fait que l'orientation nucléaire des atomes portés par la décharge dans divers niveaux excités est partiellement transformée en orientation électronique sous l'effet du couplage hyperfin; les signaux de détection enregistrés reflètent ainsi directement l'orientation des atomes de ${ }^{3} \mathrm{He}$ dans le niveau fondamental.

La figure 2 schématise un montage typique de pompage optique dans ${ }^{3} \mathrm{He}$; on applique suivant la direction $O z$ du faisceau de pompage un champ magnétique $\mathbf{B}_{0}$; l'adjonction d'un champ oscillant

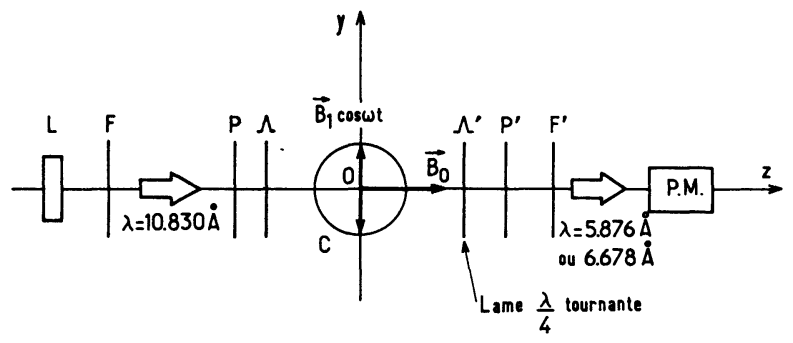

FIG. 2. - Schéma du dispositif expérimental de pompage optique dans ${ }^{3} \mathrm{He}$. Un faisceau de pompage $(\lambda=10830 \AA)$, issu d'une lampe $\mathrm{L}$, traverse un filtre infra-rouge $\mathrm{F}$, un polariseur linéaire $\mathrm{P}$, une lame quart d'onde $\Lambda$ et tombe sur la cellule de résonance $\mathrm{C}$ dans laquelle est entretenue une décharge faible. La détection utilise les raies émises par cette décharge $(\lambda=5876 \AA$ ou $\lambda=6678 \AA \AA$ ) ; le faisceau de détection traverse successivement : une lame quart d'onde tournante $\Lambda^{\prime}$, un polariseur $\mathrm{P}^{\prime}$, un filtre interférentiel $F^{\prime}$ et tombe sur un photomultiplicateur PM.

$\mathbf{B}_{1} \cos \omega t$, perpendiculaire à $\mathbf{B}_{0}$, permet d'effectuer des expériences de résonance magnétique; on détecte l'orientation nucléaire longitudinale $\left\langle I_{z}\right\rangle$ en mesurant la polarisation de la lumière émise par la décharge dans la direction $\mathrm{Oz}$.
1. Equation d'évolution des observables atomiques. Dans cette partie, nous établissons les équations couplées qui donnent l'évolution des atomes de ${ }^{3} \mathrm{He}$ dans le niveau fondamental et le niveau métastable au cours d'une expérience de pompage optique. Nous définissons d'abord l'ensemble des observables, dont les valeurs moyennes permettent de caractériser l'état angulaire des atomes dans le niveau fondamental $1^{1} \mathrm{~S}_{0}$ et dans les deux sous-niveaux hyperfins du métastable $2{ }^{3} \mathrm{~S}_{1}$. Nous déterminons ensuite l'effet des collisions d'échange de métastabilité sur ces observables, puis nous envisageons les autres causes d'évolution intervenant dans une telle expérience.

1.1 Définition Des ObSERVAbles. - Soit $\rho_{\mathrm{f}}$ la matrice densité des atomes dans l'état fondamental; $\rho_{\mathrm{f}}$ peut être caractérisé par la donnée des trois composantes de l'orientation nucléaire :

$$
\langle\mathbf{I}\rangle_{\mathrm{f}}=\operatorname{Tr}\left\{\rho_{\mathrm{f}} \mathbf{I}\right\} .
$$

Appelons $\rho_{\mathrm{m}}$ la matrice densité des atomes dans le niveau métastable $2{ }^{3} \mathrm{~S}_{1}, P_{3 / 2}$ et $P_{1 / 2}$ les projecteurs sur les sous-niveaux hyperfins $F=\frac{3}{2}$ et $F=\frac{1}{2}$. Dans le niveau $2{ }^{3} \mathrm{~S}_{1}$, le moment cinétique orbital des électrons est nul, de sorte que le moment cinétique total est donné par :

$$
\mathbf{F}=\mathbf{I}+\mathbf{S}
$$

où $\mathbf{S}$ est le moment cinétique total de spin électronique; on montre aisément que $P_{3 / 2}$ et $P_{1 / 2}$ peuvent s'exprimer sous la forme :

$$
\left\{\begin{array}{l}
P_{3 / 2}=\frac{2}{3}+\frac{2}{3} \text { I.S } \\
P_{1 / 2}=\frac{1}{3}-\frac{2}{3} \text { I.S }
\end{array}\right.
$$

Les valeurs moyennes :

$$
\begin{aligned}
& \left\langle P_{3 / 2}\right\rangle=\operatorname{Tr}\left\{\rho_{\mathrm{m}} P_{3 / 2}\right\} \\
& \left\langle P_{1 / 2}\right\rangle=\operatorname{Tr}\left\{\rho_{\mathrm{m}} P_{1 / 2}\right\}
\end{aligned}
$$

donnent les populations des deux sous-niveaux hyperfins; la quantité

$$
D=\frac{1}{4} P_{3 / 2}-\frac{1}{2} P_{1 / 2}
$$

a, d'après (1.3), pour valeur moyenne :

$$
\langle D\rangle=-\frac{1}{6}\langle\mathbf{I} . \mathbf{S}\rangle=-\frac{1}{6} \operatorname{Tr}\left\{\rho_{\mathrm{m}} \text { I.S }\right\}
$$

$\langle D\rangle$ est appelée « différence de populations hyperfines » : elle est nulle si $\rho_{\mathrm{m}}$ est proportionnelle à la matrice unité, et caractérise l'écart des populations hyperfines par rapport à l'équilibre.

Les orientations de chacun des sous-niveaux hyperfins valent :

$$
\left\{\begin{array}{l}
\langle\mathbf{F}\rangle_{3 / 2}=\operatorname{Tr}\left\{P_{3 / 2} \rho_{\mathrm{m}} P_{3 / 2} \mathbf{F}\right\} \\
\langle\mathbf{F}\rangle_{1 / 2}=\operatorname{Tr}\left\{P_{1 / 2} \rho_{\mathrm{m}} P_{1 / 2} \mathbf{F}\right\} .
\end{array}\right.
$$


Il ne peut exister d'alignement dans le sous-niveau $F=\frac{1}{2}$; quant au niveau $F=\frac{3}{2}$, son alignement peut être caractérisé par les valeurs moyennes :

avec :

$$
\left\langle K_{\alpha \beta}\right\rangle_{\mathrm{m}}=\operatorname{Tr}\left\{P_{3 / 2} \rho_{\mathrm{m}} P_{3 / 2} K_{\alpha \beta}\right\}
$$

$$
K_{\alpha \beta}=3 \frac{F_{\alpha} F_{\beta}+F_{\beta} F_{\alpha}}{2}-\mathbf{F}^{2} \delta_{\alpha \beta}
$$

(où $\alpha, \beta=x, y$ ou $z$ ). Enfin, on pourrait définir dans le niveau $F=\frac{3}{2}$ un opérateur tensoriel d'ordre 3; toutefois, on ne peut pas détecter optiquement une telle observable et, de plus, nous verrons que son évolution n'est pas couplée à celles des grandeurs qui nous intéressent; nous n'en parlerons donc pas.

Nous avons ainsi envisagé l'ensemble des observables du niveau métastable qui concernent soit le sous-niveau $F=\frac{3}{2}$, soit le sous-niveau $F=\frac{1}{2}$; leurs valeurs moyennes permettent de caractériser les populations et les cohérences Zeeman de $\rho_{\mathrm{m}}$. Bien sûr, $\rho_{\mathrm{m}}$ peut a priori avoir également des cohérences hyperfines, qu'il faudrait caractériser par des observables d'ordre 1 ou 2 ; nous verrons cependant que, dans les expériences envisagées, ces cohérences hyperfines restent constamment nulles.

Il nous sera utile pour la suite de considérer les orientations électronique ou nucléaire du niveau métastable, que nous noterons respectivement $\langle\mathbf{I}\rangle_{\mathrm{m}}$ et $\langle\mathbf{S}\rangle_{\mathrm{m}}$. En l'absence de cohérences hyperfines, ces deux valeurs moyennes s'expriment en fonction de $\langle\mathbf{F}\rangle_{3 / 2}$ et $\langle\mathbf{F}\rangle_{1 / 2}$; on trouve facilement, en utilisant le théorème de projection :

$$
\left\{\begin{array}{l}
\langle\mathbf{S}\rangle_{\mathrm{m}}=\frac{2}{3}\left[\langle\mathbf{F}\rangle_{3 / 2}+2\langle\mathbf{F}\rangle_{1 / 2}\right] \\
\langle\mathbf{I}\rangle_{\mathrm{m}}=\frac{1}{3}\left[\langle\mathbf{F}\rangle_{3 / 2}-\langle\mathbf{F}\rangle_{1 / 2}\right] .
\end{array}\right.
$$

De même, il est commode d'introduire les composantes de l'alignement électronique :

$$
Q_{\alpha \beta}=3 \frac{S_{\alpha} S_{\beta}+S_{\beta} S_{\alpha}}{2}-\mathbf{S}^{2} \delta_{\alpha \beta}
$$

et celles d'un alignement « mixte » :

$$
T_{\alpha \beta}=3 \frac{I_{\alpha} S_{\beta}+I_{\beta} S_{\alpha}}{2}-\text { I.S } \delta_{\alpha \beta} .
$$

En portant l'égalité $\mathbf{F}=\mathbf{I}+\mathbf{S}$ dans la définition (1.8) de $K_{\alpha \beta}$, on obtient immédiatement :

$$
K_{\alpha \beta}=Q_{\alpha \beta}+2 T_{\alpha \beta} .
$$

On peut enfin remarquer que $K_{\alpha \beta}$ est un opérateur qui commute avec $\mathbf{F}^{2}$ et n'a donc pas d'éléments de matrice entre les sous-niveaux $F=\frac{3}{2}$ et $F=\frac{1}{2}$; en fait, $K_{\alpha \beta}$ n'a d'éléments qu'à l'intérieur du sousniveau $F=\frac{3}{2}$ [les deux projecteurs $P_{3 / 2}$ dans (1.7) sont en réalité inutiles]. Le théorème de WignerEckart permet donc d'écrire :

$$
P_{3 / 2} Q_{\alpha \beta} P_{3 / 2}=\lambda K_{\alpha \beta}
$$

où $\lambda$ est une constante; cette dernière peut aisément être calculée en reportant (1.12) dans (1.13), et en prenant les éléments de matrice de l'égalité obtenue lorsque $\alpha=\beta=z$ entre le bra $\left\langle m_{\mathrm{I}}=\frac{1}{2}, m_{\mathrm{J}}=1\right.$ । et le ket correspondant; on trouve $\lambda=\frac{1}{3}$. Pour finir, en combinant (1.12) et (1.13), on obtient :

$$
P_{3 / 2} Q_{\alpha \beta} P_{3 / 2}=\frac{1}{3}\left(Q_{\alpha \beta}+2 T_{\alpha \beta}\right)
$$

égalité qui nous sera utile dans la suite.

1.2 EFFET DE L'ÉCHANGE DE MÉTASTABILITÉ. 1.2.1 Evolution des matrices densité. - L'effet des collisions d'échange de métastabilité sur les matrices densité $\rho_{\mathrm{f}}$ et $\rho_{\mathrm{m}}$ a été étudié par Partridge et Series [19] ; rappelons brièvement leurs résultats. Supposons que deux atomes, l'un dans l'état fondamental et de matrice densité $\rho_{\mathrm{f}}$, l'autre dans l'état métastable et de matrice densité $\rho_{\mathrm{m}}$, subissent une collision d'échange de métastabilité ; immédiatement après cette collision, la matrice densité de l'atome dans l'état fondamental est :

$$
\rho_{\mathrm{f}}^{\prime}=\operatorname{Tr}_{\mathrm{e}} \rho_{\mathrm{m}}
$$

et celle de l'atome dans l'état métastable est :

$$
\rho_{\mathrm{m}}^{\prime}=\rho_{\mathrm{f}} \otimes \operatorname{Tr}_{\mathrm{n}} \rho_{\mathrm{m}}
$$

où $\operatorname{Tr}_{\mathrm{e}}$ et $\operatorname{Tr}_{\mathrm{n}}$ désignent les opérations de traces partielles respectivement sur les variables électroniques et nucléaires. Le sens physique de ces équations est clair : elles expriment que l'échange de métastabilité se traduit par un transfert pur et simple de l'état électronique métastable d'un noyau à l'autre, sans que ce transfert apporte de modification aux variables de spin des électrons et des noyaux $\left({ }^{1}\right)$. On peut remarquer que, juste après la collision, les variables nucléaires et électroniques de l'atome métastable ne présentent aucune corrélation, et que la matrice densité $\rho_{\mathrm{m}}^{\prime}$ de l'atome métastable possède des éléments de matrice non diagonaux entre les sous-niveaux $F=\frac{3}{2}$ et $F=\frac{1}{2}$ (cohérences hyperfines). Nous verrons cependant que ces éléments non diagonaux ne jouent en fait aucun rôle.

Considérons maintenant un ensemble d'atomes d'hélium, les uns dans le niveau fondamental, les autres dans le niveau métastable (rappelons que les densités $N$ d'atomes fondamentaux et $n$ d'atomes métastables sont, en pratique, dans un rapport de l'ordre de $10^{6}$ ). Soit $1 / \tau$ la probabilité par unité de temps pour qu'un atome métastable donné subisse une collision d'échange avec un des atomes dans l'état fondamental. Pendant un intervalle de temps $\mathrm{d} t$,

( $\left.{ }^{1}\right)$ Ceci résulte à la fois du caractère électrostatique de l'interaction responsable de l'échange de métastabilité, et de la brièveté de la collision. D'un point de vue expérimental, le fait que les collisions d'échange de métastabilité dans un gaz de ${ }^{4} \mathrm{He}$ pur ne sont la cause d'aucun élargissement de la résonance magnétique dans le niveau $2{ }^{3} \mathrm{~S}_{1}$, permet de vérifier de façon très sensible que les variables électroniques ne sont pas affectées par ces collisions. 
il y a une proportion $\mathrm{d} t / \tau$ des atomes métastables qui subissent une telle collision, ce qui change leur matrice densité $\rho_{\mathrm{m}}$ en $\rho_{\mathrm{m}}^{\prime}$; l'évolution de la matrice densité de l'ensemble des atomes métastables est alors donnée par :

$$
\mathrm{d} \rho_{\mathrm{m}}=-\frac{\mathrm{d} t}{\tau} \rho_{\mathrm{m}}+\frac{\mathrm{d} t}{\tau} \rho_{\mathrm{m}}^{\prime}
$$

Bien sûr, pendant ce même intervalle de temps $\mathrm{d} t$, un nombre égal $n \frac{\mathrm{d} t}{\tau}$ (par unité de volume) d'atomes fondamentaux a subi une collision d'échange de métastabilité; comme ces atomes représentent une proportion $\frac{n}{N} \frac{\mathrm{d} t}{\tau} \mathrm{du}$ nombre total d'atomes fondamentaux, l'évolution correspondante de la matrice densité $\rho_{\mathrm{f}}$ de l'ensemble des atomes s'écrit :

$$
\mathrm{d} \rho_{\mathrm{f}}=-\frac{\mathrm{d} t}{T} \rho_{\mathrm{f}}+\frac{\mathrm{d} t}{T} \rho_{\mathrm{f}}^{\prime}
$$

où la probabilité par unité de temps $1 / T$ d'échange pour un atome fondamental est donnée par :

$$
\frac{1}{T}=\frac{n}{N} \frac{1}{\tau}
$$

Des valeurs typiques pour les temps d'échange sont $\tau \simeq 10^{-7} \mathrm{~s}$ et $T \simeq 10^{-1} \mathrm{~s}$ (voir Tableau $\mathrm{I}$ )

\section{TABLEAU 1}

Ordres de grandeur typiques des différentes constantes de temps caractéristiques des niveaux fonda-

\begin{tabular}{|c|c|c|}
\hline $\begin{array}{l}\text { Niveau } \\
\text { fondamental }\end{array}$ & $\left\{\begin{array}{l}\text { temps d'échange de métastabilité } \\
\text { temps de relaxation longitudinal } \\
\text { (dans la décharge) }\end{array}\right.$ & $\begin{array}{l}T \\
T_{\mathrm{r}}\end{array}$ \\
\hline 18 & $\begin{array}{l}\text { temps d'échange } \\
\text { temps de relaxation }\end{array}$ & $\begin{array}{c}\tau \\
\tau_{\boldsymbol{r}}\end{array}$ \\
\hline étas & $\begin{array}{l}\text { temps de «pompage " des deux } \\
\text { sous-niveaux hyperfins }\end{array}$ & $\begin{array}{c}\tau_{\mathbf{p}} \\
\tau_{\mathbf{p}}^{\prime}\end{array}$ \\
\hline
\end{tabular}
mental et métastable, pour une expérience de pompage optique de ${ }^{3} \mathrm{He}$ à température ordinaire.

Nous avons mentionné plus haut que, juste après collision, la matrice densité $\rho_{\mathrm{m}}^{\prime}$ des atomes métastables possède des cohérences hyperfines, même si $\rho_{\mathrm{m}}$ n'en comporte pas : l'échange de métastabilité couple donc les populations et cohérences Zeeman aux cohérences hyperfines. Cependant, un tel couplage est non séculaire, et il ne joue en fait aucun rôle. En effet, les fréquences propres des cohérences hyperfines sont très proches de $\Delta W \simeq 6,6 \mathrm{GHz}$ (fréquence associée à la structure hyperfine du niveau $2{ }^{3} \mathrm{~S}_{1}$ ), alors que les populations et cohérences de Zeeman évoluent beaucoup plus lentement (on suppose que $\mathbf{B}_{0}$ n'est pas assez intense pour produire un découplage hyperfin dans le niveau $2{ }^{3} \mathrm{~S}_{1}$ ). L'intensité du couplage entre populations et cohérences hyperfines étant caractérisée par la quantité $1 / \tau$, ce couplage sera non séculaire si :

$$
\Delta W . \tau \gg 1
$$

Or, dans les expériences de pompage optique, où la pression d'hélium est de l'ordre du torr, cette condition est très largement réalisée $\left({ }^{2}\right)$. On peut donc considérer l'évolution des populations et cohérences Zeeman comme totalement découplées de celle des cohérences hyperfines, ce qui permet de complètement séparer dans les matrices densité $\rho_{\mathrm{m}}$ et $\rho_{\mathrm{m}}^{\prime}$ les parties qui concernent ces cohérences hyperfines et celles qui ne les concernent pas. Dans cet article, nous ne nous intéresserons pas aux cohérences hyperfines (elles pourraient par exemple être créées si l'on effectuait une résonance magnétique à la fréquence $\Delta W$, mais nous n'envisagerons pas ici une telle expérience); c'est pourquoi nous supposerons que $\rho_{\mathrm{m}}$ n'en possède pas, et nous supprimons celles qui apparaissent dans $\rho_{\mathrm{m}}^{\prime}$. Ceci revient à remplacer dans (1.17) $\rho_{\mathrm{m}}^{\prime} \operatorname{par} \rho_{\mathrm{m}}^{\prime \prime}\left({ }^{3}\right)$ :

$$
\rho_{\mathrm{m}}^{\prime \prime}=\sum_{F=1 / 2,3 / 2} P_{F} \rho_{\mathrm{m}}^{\prime} P_{F} .
$$

Par suite, les éq. (1.17) et (1.18) deviennent, compte tenu de $(1.15)$ et $(1.16)$ :

$\frac{\mathrm{d}}{\mathrm{d} t} \rho_{\mathrm{f}}=\frac{1}{T}\left[-\rho_{\mathrm{f}}+\operatorname{Tr}_{\mathrm{e}} \rho_{\mathrm{m}}\right]$,

$\frac{\mathrm{d}}{\mathrm{d} t} \rho_{\mathrm{m}}=\frac{1}{\tau}\left[-\rho_{\mathrm{m}}+\sum_{F=1 / 2,3 / 2} P_{F}\left(\rho_{\mathrm{f}} \otimes \operatorname{Tr}_{\mathrm{n}} \rho_{\mathrm{m}}\right) P_{F}\right]$.

Ces égalités sont les égalités (5) de la référence [19].

( ${ }^{2}$ A 1 torr, on trouve $\Delta W \cdot \tau \simeq 10^{3}$; il faudrait une pression de plusieurs atmosphères pour rendre les collisions d'échange assez fréquentes pour que $\Delta W . \tau \simeq 1$.

$\left({ }^{3}\right)$ D'un point de vue physique, on peut comprendre de la façon suivante pourquoi il est possible de remplacer simplement $\rho_{\mathrm{m}}^{\prime}$ par $\rho_{\mathrm{m}}^{\prime \prime}$. Pendant un intervalle de temps infinitésimal $\mathrm{d} t$, la variation de $\rho_{\mathrm{m}}$ est donnée par (1.16) et (1.17) : les variations de $\rho_{\mathrm{m}}$ sont aussi importantes pour les cohérences hyperfines que pour les populations et cohérences Zeeman. Cependant, considérons la variation de $\rho_{\mathrm{m}}$ sur un temps $\Delta t$ court devant $\tau$, mais long devant $1 / \Delta W$ (ce qui est possible d'après $(1.20)$ ); les variations des cohérences hyperfines deviennent alors totalement négligeables. En effet, si un atome donné subit une collision d'échange de métastabilité, il acquiert des cohérences hyperfines dont la phase, initialement donnée par (1.16), évolue ensuite à la fréquence $\Delta W$. Prenons maintenant un autre atome subissant une collision d'échange un temps $\frac{1}{2} \Delta W$ plus tard; la phase initiale de ses cohérences étant encore donnée par (1.16), elles annuleront exactement celles du premier atome (comme $\Delta t \ll \tau$, ce premier atome n'a pas subi entre temps de seconde collision d'échange). Pour un ensemble d'atomes subissant, à des instants aléatoires, des collisions d'échange qui créent toutes des cohérences hyperfines de même module et même phase, on conçoit donc que l'évolution rapide de cette phase à la fréquence $\Delta W$ annule les cohérences hyperfines de la matrice densité globale. Pour finir, tant que l'on ne considère que l'évolution des atomes sur des temps longs devant $1 / \Delta W$, on peut remplacer $\rho_{\mathrm{m}}^{\prime}$ par $\rho_{\mathrm{m}}^{\prime \prime}$ ce qui conduit à l'égalité $(1.22 b)$. Il ne faut pas confondre cette disparition des cohérences hyperfines, qui prend un temps de l'ordre de $1 / \Delta W$, avec l'amortissement que produirait une relaxation due par exemple à des collisions (nous n'avons pas à ce stade introduit de telle relaxation). Il faut plutôt considérer que le brouillage des cohérences hyperfines (produit par l'hamiltonien hyperfin) est analogue au brouillage des cohérences Zeeman (produit par l'hamiltonien Zeeman) qui explique l'effet Hanle. 
1.2.2 Evolution des observables. - L'effet de l'échange de métastabilité sur la valeur moyenne d'une observable $M$, relative au niveau fondamental ou au niveau métastable, s'obtient en multipliant par $M$ l'une des deux équations (1.22), puis en prenant la trace des deux membres

๙) Niveau fondamental. - L'éq. (1.22a) donne :

$$
\begin{aligned}
\frac{\mathrm{d}}{\mathrm{d} t}\langle\mathbf{I}\rangle_{\mathrm{f}} & =\operatorname{Tr}_{\mathrm{n}}\left\{\mathbf{I} \frac{\mathrm{d}}{\mathrm{d} t} \rho_{\mathrm{f}}\right\} \\
& =-\frac{1}{T}\langle\mathbf{I}\rangle_{\mathrm{f}}+\frac{1}{T} \operatorname{Tr}_{\mathrm{n}}\left\{\mathbf{I} \operatorname{Tr}_{\mathrm{e}} \rho_{\mathrm{m}}\right\}
\end{aligned}
$$

c'est-à-dire :

$$
\frac{\mathrm{d}}{\mathrm{d} t}\langle\mathbf{I}\rangle_{\mathrm{f}}=-\frac{1}{T}\langle\mathbf{I}\rangle_{\mathrm{f}}+\frac{1}{T}\langle\mathbf{I}\rangle_{\mathrm{m}} .
$$

Cette égalité exprime simplement que les collisions d'échange de métastabilité tendent à remplacer l'orientation nucléaire du fondamental par celle du niveau métastable. On peut d'ailleurs reporter $(1.9 b)$ dans (1.24) pour mettre en évidence la façon dont les orientations des deux sous-niveaux hyperfins $F=\frac{3}{2}$ et $F=\frac{1}{2}$ contribuent à ce transfert d'orientation :

$\frac{\mathrm{d}}{\mathrm{d} t}\langle\mathbf{I}\rangle_{\mathrm{f}}=-\frac{1}{T}\langle\mathbf{I}\rangle_{\mathrm{f}}+\frac{1}{3 T}\langle\mathbf{F}\rangle_{3 / 2}-\frac{1}{3 T}\langle\mathbf{F}\rangle_{1 / 2}$.

On constate alors que les orientations $\langle\mathbf{F}\rangle_{3 / 2}$ et $\langle\mathbf{F}\rangle_{1 / 2}$ sont transférées avec des signes opposés; physiquement, ceci provient de ce que $\langle\mathbf{I}\rangle$ et $\langle\mathbf{F}\rangle$ sont parallèles dans le sous-niveau $F=\frac{3}{2}$, antiparallèles dans le sous-niveau $F=\frac{1}{2}$.

ß) Niveau métastable. - (i) Populations des sousniveaux hyperfins. - En multipliant $(1.22 b)$ par $P_{F}$ et en prenant la trace des deux membres, on obtient :

$\frac{\mathrm{d}}{\mathrm{d} t}\left\langle P_{F}\right\rangle=-\frac{1}{\tau}\left\langle P_{F}\right\rangle+\frac{1}{\tau} \operatorname{Tr}\left\{P_{F}\left(\rho_{\mathrm{f}} \otimes \operatorname{Tr}_{\mathrm{n}} \rho_{\mathrm{m}}\right) P_{F}\right\}$.

Dans le dernier terme de cette équation, on peut supprimer un des projecteurs $P_{F}$ (il suffit par exemple d'effectuer une permutation circulaire des opérateurs pour faire apparaître $P_{F}^{2}=P_{F}$ ), et remplacer $P_{F}$ par ses expressions (1.3); il vient alors :

$\left\{\begin{array}{rr}\frac{\mathrm{d}}{\mathrm{d} t}\left\langle P_{3 / 2}\right\rangle=-\frac{1}{\tau}\left\langle P_{3 / 2}\right\rangle+\frac{2}{3 \tau}\left(1+\langle\mathbf{I}\rangle_{\mathrm{f}} \cdot\langle\mathbf{S}\rangle_{\mathrm{m}}\right) \\ \quad(1.27 a) \\ \frac{\mathrm{d}}{\mathrm{d} t}\left\langle P_{1 / 2}\right\rangle=-\frac{1}{\tau}\left\langle P_{1 / 2}\right\rangle+\frac{1}{3 \tau}\left(1-2\langle\mathbf{I}\rangle_{\mathrm{f}} \cdot\langle\mathbf{S}\rangle_{\mathrm{m}}\right) .\end{array}\right.$

On vérifie immédiatement que

$$
\frac{\mathrm{d}}{\mathrm{d} t}\left(\left\langle P_{3 / 2}\right\rangle+\left\langle P_{1 / 2}\right\rangle\right)=0 ;
$$

en outre, la différence de populations hyperfines obéit à l'équation :

$\frac{\mathrm{d}}{\mathrm{d} t}\langle D\rangle=-\frac{1}{\tau}\langle D\rangle+\frac{1}{2 \tau}\langle\mathbf{I}\rangle_{\mathrm{f}} \cdot\langle\mathbf{S}\rangle_{\mathrm{m}}$.

Suivant que $\langle\mathbf{I}\rangle_{\mathrm{f}}$ et $\langle\mathbf{S}\rangle_{\mathrm{m}}$ sont de même sens, ou de sens opposé, le déséquilibre des populations tend à se faire au profit du sous-niveau $F=\frac{3}{2}$ ou $F=\frac{1}{2}$.

(ii) Les orientations. - D'après (1.22b), l'évolution de l'orientation dans le sous-niveau hyperfin $F$ sous l'effet de l'échange de métastabilité s'écrit :

$\frac{\mathrm{d}}{\mathrm{d} t}\langle\mathbf{F}\rangle_{\boldsymbol{F}}=-\frac{1}{\tau}\langle\mathbf{F}\rangle_{F}+\frac{1}{\tau} \operatorname{Tr}\left\{\mathbf{F} P_{F}\left(\rho_{\mathrm{f}} \otimes \operatorname{Tr}_{\mathrm{n}} \rho_{\mathrm{m}}\right) P_{F}\right\}$.

Or $P_{F}$ commute avec $\mathrm{F}$, et son carré est $P_{F}^{2}=P_{F}$; on est donc ramené au calcul de la quantité

$$
\operatorname{Tr}\left\{\mathbf{F} P_{\boldsymbol{F}}\left(\rho_{\mathrm{f}} \otimes \operatorname{Tr}_{\mathrm{n}} \rho_{\mathrm{m}}\right)\right\} .
$$

Pour cela, utilisons les égalités, déduites de (1.3) :

$$
\left\{\begin{array}{l}
F_{\alpha} P_{3 / 2}=\left(I_{\alpha}+S_{\alpha}\right)\left(\frac{2}{3}+\frac{2}{3} \mathbf{I} . \mathbf{S}\right) \\
F_{\alpha} P_{1 / 2}=\left(I_{\alpha}+S_{\alpha}\right)\left(\frac{1}{3}-\frac{2}{3} \mathbf{I} . \mathbf{S}\right)
\end{array}\right.
$$

qui permettent d'écrire :

$$
\begin{array}{r}
\operatorname{Tr}\left\{F_{\alpha} P_{3 / 2} \rho_{\mathrm{f}} \otimes \operatorname{Tr}_{\mathrm{n}} \rho_{\mathrm{m}}\right\}=\frac{2}{3}\left[\left\langle I_{\alpha}\right\rangle_{\mathrm{f}}+\left\langle S_{\alpha}\right\rangle_{\mathrm{m}}\right]+ \\
\quad+\frac{2}{3} \sum_{\beta}\left[\left\langle I_{\alpha} I_{\beta}\right\rangle_{\mathrm{f}}\left\langle S_{\beta}\right\rangle_{\mathrm{m}}+\left\langle S_{\alpha} S_{\beta}\right\rangle_{\mathrm{m}}\left\langle I_{\beta}\right\rangle_{\mathrm{f}}\right] .
\end{array}
$$

Comme le spin $I$ vaut $\frac{1}{2}$, on a :

$$
I_{\alpha} I_{\beta}=\frac{i}{2} \varepsilon_{\alpha \beta \gamma} I_{\gamma}+\frac{1}{4} \delta_{\alpha \beta}
$$

(les $\varepsilon_{\alpha \beta \gamma}$ valent +1 si $\alpha, \beta, \gamma$ forment une permutation paire de $x, y, z,-1$ si la permutation est impaire, et zéro si deux indices des $\alpha, \beta, \gamma$ sont les mêmes). D'autre part, il découle immédiatement de la définition (1.10) du tenseur $\mathbf{Q}$ ainsi que des relations de commutation de $S_{\alpha}$ et $S_{\beta}$ que :

$$
S_{\alpha} S_{\beta}=\frac{i}{2} \varepsilon_{\alpha \beta \gamma} S_{\gamma}+\frac{1}{3} Q_{\alpha \beta}+\frac{1}{3} \mathbf{S}^{2} \delta_{\alpha \beta} .
$$

Il vient par suite :

$$
\begin{aligned}
& \sum_{\beta}\left[\left\langle I_{\alpha} I_{\beta}\right\rangle_{\mathrm{f}}\left\langle S_{\beta}\right\rangle_{\mathrm{m}}+\left\langle S_{\alpha} S_{\beta}\right\rangle_{\mathrm{m}}\left\langle I_{\beta}\right\rangle_{\mathrm{f}}\right]= \\
& =\frac{1}{4}\left\langle S_{\alpha}\right\rangle_{\mathrm{m}}+\frac{2}{3}\left\langle I_{\alpha}\right\rangle_{\mathrm{f}}+\frac{1}{3} \sum_{\beta}\left\langle Q_{\alpha \beta}\right\rangle_{\mathrm{m}}\left\langle I_{\beta}\right\rangle_{\mathrm{f}} .
\end{aligned}
$$

Pour finir, l'évolution de $\langle\mathbf{F}\rangle_{3 / 2}$ est donnée par :

$$
\begin{gathered}
\frac{\mathrm{d}}{\mathrm{d} t}\langle\mathbf{F}\rangle_{3 / 2}=-\frac{1}{\tau}\langle\mathbf{F}\rangle_{3 / 2}+\frac{5}{6 \tau}\langle\mathbf{S}\rangle_{\mathrm{m}}+ \\
+\frac{10}{9 \tau}\langle\mathbf{I}\rangle_{\mathrm{f}}+\frac{2}{9 \tau}\langle\mathbf{Q}\rangle_{\mathrm{m}}\langle\mathbf{I}\rangle_{\mathrm{f}} .
\end{gathered}
$$


En procédant de la même façon, on obtient pour $\langle\mathbf{F}\rangle_{1 / 2}$ :

$$
\begin{gathered}
\frac{\mathrm{d}}{\mathrm{d} t}\langle\mathbf{F}\rangle_{1 / 2}=-\frac{1}{\tau}\langle\mathbf{F}\rangle_{1 / 2}+\frac{1}{6 \tau}\langle\mathbf{S}\rangle_{\mathrm{m}}- \\
-\frac{1}{9 \tau}\langle\mathbf{I}\rangle_{\mathrm{f}}-\frac{2}{9 \tau}\langle\mathbf{Q}\rangle_{\mathrm{m}}\langle\mathbf{I}\rangle_{\mathrm{f}} .
\end{gathered}
$$

$\mathrm{Au}$ second membre de chacune des deux éq. (1.35) on trouve un terme à coefficient négatif, qui exprime la disparition de l'orientation du métastable entrant dans la collision, suivi de trois termes décrivant l'orientation qui subsiste pour le métastable sortant. Cette dernière provient de trois sources :

- de l'orientation électronique apportée par l'atome métastable entrant dans la collision (terme en $\langle\mathbf{S}\rangle_{\mathrm{m}}$ );

- de l'orientation nucléaire transférée à partir de l'atome dans le niveau fondamental (terme en $\langle\mathbf{I}\rangle_{\mathrm{f}}$ );

- du couplage entre cette orientation nucléaire et l'alignement électronique du métastable (terme en $\langle\mathbf{Q}\rangle_{\mathrm{m}}\langle\mathbf{I}\rangle_{\mathrm{r}}$ ).

On peut remarquer qu'en multipliant (1.25) par $N$, $(1.35 a)$ et $(1.35 b)$ par $n$ et en effectuant la somme membre à membre, on trouve :

$$
\frac{\mathrm{d}}{\mathrm{d} t}\left[N\langle\mathbf{I}\rangle_{\mathrm{f}}+n\langle\mathbf{F}\rangle_{3 / 2}+n\langle\mathbf{F}\rangle_{1 / 2}\right]=0
$$

relation qui exprime simplement la conservation du moment cinétique de spin de l'ensemble des atomes au cours des collisions d'échange de métastabilité.

En utilisant les égalités $(1.9 a)$ et $(1.9 b)$, on peut mettre les éq. (1.35) sous la forme :

$$
\begin{gathered}
\frac{\mathrm{d}}{\mathrm{d} t}\langle\mathbf{F}\rangle_{3 / 2}=-\frac{4}{9 \tau}\langle\mathbf{F}\rangle_{3 / 2}+\frac{10}{9 \tau}\langle\mathbf{F}\rangle_{1 / 2}+ \\
+\frac{10}{9 \tau}\langle\mathbf{I}\rangle_{\mathrm{f}}+\frac{2}{9 \tau}\langle\mathbf{Q}\rangle_{\mathrm{m}}\langle\mathbf{I}\rangle_{\mathrm{f}}, \\
\frac{\mathrm{d}}{\mathrm{d} t}\langle\mathbf{F}\rangle_{1 / 2}=\frac{1}{9 \tau}\langle\mathbf{F}\rangle_{3 / 2}-\frac{7}{9 \tau}\langle\mathbf{F}\rangle_{1 / 2}- \\
-\frac{1}{9 \tau}\langle\mathbf{I}\rangle_{\mathrm{f}}-\frac{2}{9 \tau}\langle\mathbf{Q}\rangle_{\mathrm{m}}\langle\mathbf{I}\rangle_{\mathrm{f}}
\end{gathered}
$$

Nous constatons ainsi que l'échange de métastabilité ne se traduit pas par une destruction totale de l'orientation de chacun des sous-niveaux $F$ du métastable à chaque collision : par exemple, au second membre de l'éq. $(1.37 a)$, on trouve un terme en $-\frac{4}{9 \tau}\langle\mathbf{F}\rangle_{3 / 2}$, au lieu du terme $-\frac{1}{\tau}\langle\mathbf{F}\rangle_{3 / 2}$ qui correspondrait à une perte complète d'orientation pour le sous-niveau $F=\frac{3}{2}$; de même, pour le sousniveau $F=\frac{1}{2}$, la perte d'orientation est réduite par un facteur $\frac{7}{9}$ (cf. 1.37b); ces facteurs $\frac{4}{9}$ et $\frac{7}{9}$ proviennent de la présence des termes en $\langle\mathbf{S}\rangle_{\mathrm{m}}$ dans les éq. (1.35) : rappelons que ceux-ci traduisent la conservation du spin électronique du métastable au cours de la colli- sion. Cette conservation de $\langle\mathbf{S}\rangle_{\mathrm{m}}$ entraîne également l'existence de transferts d'orientation entre les deux sous-niveaux hyperfins (terme $\frac{10}{9 \tau}\langle\mathbf{F}\rangle_{1 / 2}$ dans $(1.37 a)$ et $\frac{1}{9 \tau}\langle\mathbf{F}\rangle_{3 / 2}$ dans $\left.(1.37 b)\right)$ : nous verrons plus loin certaines conséquences de ce couplage. Enfin, de même que les équations d'évolution des populations hyperfines font intervenir les orientations $\langle\mathbf{I}\rangle_{\mathrm{f}}$ et $\langle\mathbf{S}\rangle_{\mathrm{m}}$, les équations des orientations font intervenir l'alignement $\langle\mathbf{Q}\rangle_{\mathrm{m}}$; pour le niveau métastable, les évolutions des observables d'ordres tensoriels différents sont donc couplées lorsqu'il existe une orientation nucléaire dans le niveau fondamental.

(iii) L'alignement. - L'évolution de l'alignement $\langle\mathbf{Q}\rangle_{\mathbf{m}}$ dans le niveau métastable est donnée, d'après $(1.22 b)$ par :

$$
\begin{aligned}
\frac{\mathrm{d}}{\mathrm{d} t}\left\langle Q_{\alpha \beta}\right\rangle_{\mathrm{m}}=-\frac{1}{\tau}\left\langle Q_{\alpha \beta}\right\rangle_{\mathrm{m}}+ \\
\quad+\frac{1}{\tau} \operatorname{Tr}\left\{Q_{\alpha \beta} \sum_{F} P_{F}\left(\rho_{\mathrm{f}} \otimes \operatorname{Tr}_{\mathrm{n}} \rho_{\mathrm{m}}\right) P_{F}\right\} .
\end{aligned}
$$

Par permutation circulaire des opérateurs dans la trace, on fait apparaître l'opérateur $\sum_{F} P_{F} Q_{\alpha \beta} P_{F}$ qui vaut simplement $P_{3 / 2} Q_{\alpha \beta} P_{3 / 2}$ (l'alignement est nul dans le niveau $F=\frac{1}{2}$ ); compte tenu de (1.14), la trace devient alors :

$$
\frac{1}{3} \operatorname{Tr}\left\{\left(Q_{\alpha \beta}+2 T_{\alpha \beta}\right)\left(\rho_{\mathrm{f}} \otimes \operatorname{Tr}_{\mathrm{n}} \rho_{\mathrm{m}}\right)\right\}
$$

et (1.38) s'écrit :

$$
\begin{aligned}
\frac{\mathrm{d}}{\mathrm{d} t}\left\langle Q_{\alpha \beta}\right\rangle_{\mathrm{m}} & =-\frac{2}{3 \tau}\left\langle Q_{\alpha \beta}\right\rangle_{\mathrm{m}}+ \\
+\frac{2}{3 \tau}\left[3 \frac{\left\langle I_{\alpha}\right\rangle_{\mathrm{f}}}{\left\langle S_{\beta}\right\rangle_{\mathrm{m}}+\left\langle I_{\beta}\right\rangle_{\mathrm{f}}\left\langle S_{\alpha}\right\rangle_{\mathrm{m}}}\right. & - \\
& \left.-\delta_{\alpha \beta}\langle\mathbf{I}\rangle_{\mathrm{f}} \cdot\langle\mathbf{S}\rangle_{\mathrm{m}}\right] .
\end{aligned}
$$

Nous voyons donc que l'alignement, comme l'orientation, n'est pas entièrement détruit au cours de chaque collision d'échange de métastabilité : le facteur $\frac{2}{3}$ de réduction qui apparaît dans (1.39) (terme $\left.-\frac{2}{3 \tau}\left\langle Q_{\alpha \beta}\right\rangle_{\mathrm{m}}\right)$ a d'ailleurs été calculé par Rosner et Pipkin dans une publication récente [16].

On pourrait s'étonner de l'absence d'opérateur tensoriel d'ordre trois au second membre de (1.39); cette absence tient au fait que l'information sur l'état angulaire du métastable est transmise par l'intermédiaire du spin électronique qui, étant un moment cinétique $S=1$, donne une valeur nulle à tout tenseur d'ordre supérieur à deux.

1.2.3 Approximation des orientations faibles. Comme nous l'avons déjà signalé, les taux de pola- 
risation obtenus dans les expériences de pompage optique sur ${ }^{3} \mathrm{He}$ sont couramment de l'ordre de 1 à $5 \%$ et, dans les meilleures conditions, toujours inférieurs à $25 \%$. Nous allons voir que ceci permet de négliger dans les éq. (1.37) les termes contenant l'alignement $\langle\mathbf{Q}\rangle$.

En effet, parmi tous les processus affectant les observables du niveau métastable $2{ }^{3} \mathrm{~S}_{1}$, l'échange de métastabilité est de loin le plus important (ceci se remarque par exemple sur le tableau I). Nous pouvons donc utiliser (1.37) et (1.39) pour calculer un ordre de grandeur des valeurs stationnaires des observables correspondantes. Négligeons d'abord dans (1.37) les termes en $\langle\boldsymbol{Q}\rangle_{\mathrm{m}}\langle\mathbf{I}\rangle_{\mathrm{f}}$ : la solution stationnaire de ces équations donne pour les taux d'orientation des niveaux $F=\frac{3}{2}$ et $\frac{1}{2}$ le même ordre de grandeur que $\langle\mathbf{I}\rangle_{\mathrm{f}}$, c'est-à-dire par exemple $10 \%$; d'après (1.39), la valeur stationnaire de $\left\langle Q_{\alpha \beta}\right\rangle_{\mathrm{m}}$ est proportionnelle à des termes du type $\left\langle I_{\alpha}\right\rangle_{\mathrm{f}}\left\langle S_{\beta}\right\rangle_{\mathrm{m}}$, ce qui entraîne que l'alignement est de l'ordre de $1 \%\left({ }^{4}\right)$. C'est pourquoi, dans les équations d'évolution des orientations, on peut négliger les termes en

$$
\langle\mathbf{Q}\rangle_{\mathrm{m}}\langle\mathbf{I}\rangle_{\mathrm{f}},
$$

qui sont approximativement 100 fois plus petits que les autres; on découple ainsi l'évolution des orientations de celle de l'alignement. Les équations obtenues en effectuant cette simplification s'écrivent :

$$
\left\{\begin{array}{lr}
\frac{\mathrm{d}}{\mathrm{d} t}\langle\mathbf{F}\rangle_{3 / 2}=-\frac{4}{9 \tau}\langle\mathbf{F}\rangle_{3 / 2}+\frac{10}{9 \tau}\langle\mathbf{F}\rangle_{1 / 2}+\frac{10}{9 \tau}\langle\mathbf{I}\rangle_{\mathrm{f}} \\
\quad(1.40 a) \\
\frac{\mathrm{d}}{\mathrm{d} t}\langle\mathbf{F}\rangle_{1 / 2}=\frac{1}{9 \tau}\langle\mathbf{F}\rangle_{3 / 2}-\frac{7}{9 \tau}\langle\mathbf{F}\rangle_{1 / 2}-\frac{1}{9 \tau}\langle\mathbf{I}\rangle_{\mathrm{f}} \\
\quad(1.40 b) \\
\frac{\mathrm{d}}{\mathrm{d} t}\langle\mathbf{I}\rangle_{\mathrm{f}}=\frac{1}{3 T}\langle\mathbf{F}\rangle_{3 / 2}-\frac{1}{3 T}\langle\mathbf{F}\rangle_{1 / 2}-\frac{1}{T}\langle\mathbf{I}\rangle_{\mathrm{f}} .
\end{array}\right.
$$

Dans la suite, nous utiliserons uniquement ces trois équations simplifiées, valables pour des taux d'orientation faibles.

1.3 Autres CAUSES D'ÉVOlution DES OBSERVABLES. - On sait qu'à température ordinaire l'échange de métastabilité est le processus dominant qui affecte l'évolution des atomes d'hélium dans les niveaux métastable et fondamental. Cependant, pour traiter complètement la cinétique du pompage optique de l'hélium dans une expérience telle que nous l'avons décrite dans les rappels (voir Fig. 2), il faut également tenir compte des autres causes d'évolution des obser-

$\left({ }^{4}\right)$ Dans les conditions ordinaires, l'alignement introduit directement par le pompage n'est guère plus important, le temps de pompage $\tau_{\mathrm{p}}$ étant toujours très grand devant $\tau$. vables atomiques; nous allons envisager successivement l'effet sur les atomes du champ magnétique statique $\mathbf{B}_{0}$, du faisceau de pompage et des processus de relaxation qui affectent les orientations des niveaux fondamental et métastable.

1.3.1 Effet des champs magnétiques. - Si l'on applique un champ magnétique statique $\mathbf{B}_{0}$ parallèle à la direction $O z$ (cf. Fig. 2), les orientations transversales des atomes précessent autour de $\mathrm{Oz}$; ceci est décrit par les équations suivantes :

$$
\begin{aligned}
& \frac{\mathrm{d}}{\mathrm{d} t}\left\langle F_{z}\right\rangle_{3 / 2}=\frac{\mathrm{d}}{\mathrm{d} t}\left\langle F_{z}\right\rangle_{1 / 2}=\frac{\mathrm{d}}{\mathrm{d} t}\left\langle I_{z}\right\rangle=0 \\
& \left\{\begin{array}{l}
\frac{\mathrm{d}}{\mathrm{d} t}\left\langle F_{+}\right\rangle_{3 / 2}=i \frac{2 \omega_{\mathrm{m}}}{3}\left\langle F_{+}\right\rangle_{3 / 2} \\
\frac{\mathrm{d}}{\mathrm{d} t}\left\langle F_{+}\right\rangle_{1 / 2}=i \frac{4 \omega_{\mathrm{m}}}{3}\left\langle F_{+}\right\rangle_{1 / 2} \\
\frac{\mathrm{d}}{\mathrm{d} t}\left\langle I_{+}\right\rangle_{\mathrm{f}}=i \omega_{\mathrm{f}}\left\langle I_{+}\right\rangle_{\mathrm{f}}
\end{array}\right.
\end{aligned}
$$

avec $I_{+}=I_{x}+i I_{y}, F_{+}=F_{x}+i F_{y} ; \omega_{\mathrm{f}}$ et $\omega_{\mathrm{m}}$ sont définis par :

$$
\left\{\begin{array}{c}
\omega_{\mathrm{f}}=-\gamma_{\mathrm{f}} B_{0} \\
\omega_{\mathrm{m}}=-\gamma_{\mathrm{m}} B_{0}
\end{array}\right.
$$

$\gamma_{\mathrm{f}}$ étant le facteur gyromagnétique du spin nucléaire dans le niveau fondamental, $\gamma_{m}$ celui du spin électronique dans le niveau métastable de ${ }^{4} \mathrm{He}\left({ }^{5}\right)$ (les facteurs $\frac{2}{3}$ et $\frac{4}{3}$ dans $(1.42 a)$ et $(1.42 b)$ proviennent des facteurs de Landé qui sont différents pour les deux sous-niveaux hyperfins $F=\frac{3}{2}$ et $F=\frac{1}{2}$ de ${ }^{3} \mathrm{He}$; nous n'avons pas tenu compte des corrections à ces facteurs de Landé liées au magnétisme nucléaire).

On peut en outre appliquer à la cellule un champ magnétique oscillant $\mathbf{B}_{1} \cos \omega t$ perpendiculaire à $\mathbf{B}_{0}$, de façon à effectuer une résonance magnétique dans l'un des niveaux atomiques. Ce cas sera envisagé au paragraphe 4 .

1.3.2 Effets du faisceau de pompage. - Ce faisceau lumineux circulairement polarisé, transmettant la raie $\lambda=10830 \AA$, se propage dans la direction $O z$ (voir Fig. 2); son effet sur les atomes métastables peut être décrit d'une façon simplifiée par les équations :

$$
\left\{\begin{array}{l}
\frac{\mathrm{d}}{\mathrm{d} t}\langle\mathbf{F}\rangle_{3 / 2}=\frac{1}{\tau_{\mathbf{p}}}\left(\boldsymbol{\Phi}-\langle\mathbf{F}\rangle_{3 / 2}\right) \\
\frac{\mathrm{d}}{\mathrm{d} t}\langle\mathbf{F}\rangle_{1 / 2}=\frac{1}{\tau_{\mathbf{p}}^{\prime}}\left(\boldsymbol{\Phi}^{\prime}-\langle\mathbf{F}\rangle_{1 / 2}\right)
\end{array}\right.
$$

$\Phi$ et $\boldsymbol{\Phi}^{\prime}$ sont deux vecteurs constants, parallèles à la direction de pompage $O z, \tau_{p}$ et $\tau_{p}^{\prime}$ des « temps de pompage » inversement proportionnels à l'intensité

$\left(^{5}\right)$ On a $\omega_{\mathrm{f}} / 2 \pi \simeq 3,243 \times B_{0} \mathrm{kHz}$ et $\omega_{\mathrm{m}} / 2 \pi \simeq 2,802 \times B_{0} \mathrm{MHz}$, $B_{0}$ étant exprimé en gauss. 
lumineuse (l'ordre de grandeur des temps $\tau_{\mathrm{p}}$ et $\tau_{\mathrm{p}}^{\prime}$ dans nos expériences est de $10^{-4} \mathrm{~s}$; voir Tableau I). En ajustant convenablement les constantes $\tau_{\mathrm{p}}, \tau_{\mathrm{p}}^{\prime}, \Phi$ et $\Phi^{\prime}$, on peut décrire l'effet de l'absorption de photons par chacun des niveaux hyperfins, qui est différent suivant que la lampe utilisée contient ${ }^{3} \mathrm{He}$ ou ${ }^{4} \mathrm{He}$ (voir rappels à la fin de l'introduction); on peut également tenir compte de la retombée de l'orientation par émission spontanée à partir du niveau $2{ }^{3} \mathrm{P}$, cette orientation étant plus ou moins détruite par les collisions, suivant la pression d'hélium [1]. Les seules approximations faites pour obtenir (1.44) sont : d'une part, on considère la relaxation optique comme isotrope (on néglige notamment tout «couplage optique » entre les orientations et l'alignement); d'autre part, on néglige le «couplage optique " entre $\langle\mathbf{F}\rangle_{3 / 2}$ et $\langle\mathbf{F}\rangle_{1 / 2}$ (l'orientation qui retombe du niveau $2{ }^{3} \mathrm{P}$ sur les deux niveaux $F=\frac{3}{2}$ et $F=\frac{1}{2}$ peut dépendre de $\langle\mathbf{F}\rangle_{3 / 2}$ et $\langle\mathbf{F}\rangle_{1 / 2}$ ). Bien que ces approximations ne soient pas essentielles pour la suite du calcul, nous nous y tiendrons pour simplifier l'exposé.

1.3.3 Processus de relaxation. - En plus des collisions d'échange de métastabilité, il existe d'autres types de collisions qui peuvent affecter les orientations des niveaux métastable et fondamental; L. D. Schearer, H. R. Byerly et W. A. Fitzsimmons ont étudié en détail les processus de relaxation qui en découlent ([20] à [23]). Pour le niveau métastable, nous décrirons leur effet par un temps de relaxation unique $\tau_{\mathrm{r}}$; ce temps est, par exemple, le temps de diffusion des métastables vers la paroi, où ils se détruisent $\left(\tau_{\mathrm{r}}\right.$ est de l'ordre de la milliseconde; $\mathrm{cf}$. Tableau I). Pour le niveau fondamental nous prendrons un temps $T_{\mathrm{r}}$, lié à la relaxation des spins nucléaires sur les parois et à l'effet de la décharge; $T_{\mathrm{r}}$ est de l'ordre de 100 secondes. On obtient ainsi :

$$
\left\{\begin{array}{l}
\frac{\mathrm{d}}{\mathrm{d} t}\langle\mathbf{F}\rangle_{F}=-\frac{1}{\tau_{\mathrm{r}}}\langle\mathbf{F}\rangle_{F} \\
\frac{\mathrm{d}}{\mathrm{d} t}\langle\mathbf{I}\rangle_{\mathrm{f}}=-\frac{1}{T_{\mathrm{r}}}\langle\mathbf{I}\rangle_{\mathrm{f}} .
\end{array}\right.
$$

1.4 POSITION DU PROBLÈME. - Les équations d'évolution globale des orientations s'obtiennent en ajoutant les différentes vitesses de variation précédemment calculées. On trouve :

$$
\begin{aligned}
& \mathrm{d}\left[\begin{array}{l}
\left\langle F_{z}\right\rangle_{3 / 2} \\
\left\langle F_{z}\right\rangle_{1 / 2} \\
\left\langle I_{z}\right\rangle_{\mathrm{f}}
\end{array}\right]=[(A)]\left[\begin{array}{l}
\left\langle F_{z}\right\rangle_{3 / 2} \\
\left\langle F_{z}\right\rangle_{1 / 2} \\
\left\langle I_{z}\right\rangle_{\mathrm{f}}
\end{array}\right]+\left[\begin{array}{c}
\Phi / \tau_{\mathrm{p}} \\
\Phi^{\prime} / \tau_{\mathrm{p}}^{\prime} \\
0
\end{array}\right] \\
& \frac{\mathrm{d}}{\mathrm{d} t}\left[\begin{array}{l}
\left\langle F_{+}\right\rangle_{3 / 2} \\
\left\langle F_{+}\right\rangle_{1 / 2} \\
\left\langle I_{+}\right\rangle_{\mathrm{f}}
\end{array}\right]=[(B)]\left[\begin{array}{l}
\left\langle F_{+}\right\rangle_{3 / 2} \\
\left\langle F_{+}\right\rangle_{1 / 2} \\
\left\langle I_{+}\right\rangle_{\mathrm{f}}
\end{array}\right]
\end{aligned}
$$

avec :

$$
[(A)]=\left[\begin{array}{ccc}
-\left(\frac{4}{9 \tau}+\frac{1}{\tau_{\mathrm{r}}}+\frac{1}{\tau_{\mathrm{p}}}\right) & \frac{10}{9 \tau} & \frac{10}{9 \tau} \\
\frac{1}{9 \tau} & -\left(\frac{7}{9 \tau}+\frac{1}{\tau_{\mathrm{r}}}+\frac{1}{\tau_{\mathrm{p}}^{\prime}}\right) & -\frac{1}{9 \tau} \\
\frac{1}{3 T} & -\frac{1}{3 T} & -\left(\frac{1}{T}+\frac{1}{T_{\mathrm{r}}}\right)
\end{array}\right]
$$

et :

$$
[(B)]=[(A)]+i\left[\begin{array}{ccc}
\frac{2 \omega_{\mathrm{m}}}{3} & 0 & 0 \\
0 & \frac{4 \omega_{\mathrm{m}}}{3} & 0 \\
0 & 0 & \omega_{\mathrm{f}}
\end{array}\right]
$$

Nous sommes donc en présence de deux systèmes de trois équations différentielles linéaires couplées; la détermination des orientations en régime stationnaire, de leurs constantes de temps d'amortissement ou des fréquences propres de leur évolution se ramène donc à l'inversion ou à la diagonalisation des deux matrices $A$ et $B$.

La valeur moyenne qui nous intéresse principalement est l'orientation nucléaire $\langle\mathbf{I}\rangle_{\mathrm{f}}$ qui, comme nous l'avons dit plus haut, est la grandeur que nous détectons dans nos expériences. C'est pourquoi, dans les calculs qui vont suivre, nous allons mettre l'accent sur l'évolution de $\left\langle I_{z}\right\rangle_{\mathrm{f}}$, sa constante de temps, l'orientation limite obtenue, ses variations lorsqu'on effectue la résonance magnétique dans l'un des sousniveaux $F=\frac{3}{2}$ ou $F=\frac{1}{2}$ ou dans le fondamental, etc... Il n'y aurait toutefois aucune difficulté à faire des calculs analogues pour $\left\langle F_{z}\right\rangle_{3 / 2}$ et $\left\langle F_{z}\right\rangle_{1 / 2}$. 
2. Cinétique du pompage optique dans. ${ }^{3} \mathrm{He}$; évolution des orientations longitudinales. - Dans ce paragraphe, nous allons utiliser les éq. (1.46) pour analyser en détail la cinétique du pompage optique de ${ }^{3} \mathrm{He}$ (constantes de temps d'évolution des orientations longitudinales des différents niveaux, orientations limites obtenues, etc...). Le mécanisme de ce pompage optique "indirect" a été étudié par Schearer et al. [1], [20] et Fitzsimmons [22]; ainsi que nous l'avons dit dans l'introduction, le point de vue global que nous adoptons ici en étudiant directement l'orientation des trois niveaux $F=\frac{3}{2}, F=\frac{1}{2}$ et fondamental permet de dégager plus clairement le sens physique des phénomènes et d'en donner une description quantitative plus complète.

Comme les références précédemment citées, nous allons tirer parti du fait que la densité $n$ d'atomes métastables est beaucoup plus faible que la densité $N$ d'atomes fondamentaux (typiquement, on a $n / N \simeq 10^{-6}$ ). Ceci entraîne que l'orientation nucléaire
$\langle\mathbf{I}\rangle_{\mathrm{f}}$ du niveau fondamental joue le rôle d'un "volant " de grande inertie, évoluant avec une constante de temps longue; ce volant d'orientation est couplé aux orientations $\langle\mathbf{F}\rangle_{3 / 2}$ et $\langle\mathbf{F}\rangle_{1 / 2}$ qui, elles, évoluent beaucoup plus rapidement : au bout d'un temps de l'ordre de $\tau$, ces observables se mettent en équilibre avec l'orientation $\langle\mathbf{I}\rangle_{\mathrm{f}}$. On s'attend donc à ce que les valeurs moyennes $\langle\mathbf{F}\rangle_{3 / 2}$ et $\langle\mathbf{F}\rangle_{1 / 2}$ suivent pratiquement sans retard l'évolution lente de $\langle\mathbf{I}\rangle_{\mathrm{f}}$. Mathématiquement, ceci se traduit par le fait qu'en mettant en facteur $1 / \tau$ dans la matrice $A$ qui figure dans (1.46), on fait apparaître certains termes qui sont de l'ordre de 1 , d'autres qui sont proportionnels à $\tau / \tau_{\mathbf{r}}, \tau / \tau_{\mathbf{p}}$, d'autres enfin à $\tau / T, \tau / T_{\mathbf{r}}$. Dans les conditions expérimentales habituelles, tous ces rapports sont petits devant 1 (cf. Tableau I) : les deux premiers sont de l'ordre de $10^{-3}$ à $10^{-4}$; quant aux deux derniers, ils sont encore beaucoup plus petits, puisqu'ils sont de l'ordre de $10^{-6}$ et $10^{-9}$ respectivement. Aussi allons-nous nous contenter de développements à l'ordre 0 ou 1 en fonction de $\tau / T$ et $\tau / T_{\text {r }}$.

2.1 CALCUl A L'ORDRE ZÉRo ; ORIENTATIONS DANS LE NIVEAU MÉTASTABLE CORRESPONDANT A UNE ORIENTATION NUCLÉAIRE DONNÉE. - 2.1.1 Les constantes de temps d'évolution. - A l'ordre zéro en $\tau / T$ et $\tau / T_{\mathrm{r}}$, la matrice $A$ s'écrit :

$$
[(A)]=\frac{1}{\tau}\left[\begin{array}{ccr}
-\left(\frac{4}{9}+\frac{\tau}{\tau_{\mathrm{r}}}+\frac{\tau}{\tau_{\mathrm{p}}}\right) & \frac{10}{9} & \frac{10}{9} \\
\frac{1}{9} & -\left(\frac{7}{9}+\frac{\tau}{\tau_{\mathrm{r}}}+\frac{\tau}{\tau_{\mathrm{p}}^{\prime}}\right) & -\frac{1}{9} \\
0 & 0 & 0
\end{array}\right] .
$$

On trouve aisément que les valeurs propres de cette matrice sont :

$$
\left\{\begin{array}{l}
a_{1} \simeq-\frac{1}{\tau}\left(\frac{2}{9}+\frac{\tau}{\tau_{\mathrm{r}}}+\frac{5}{7} \frac{\tau}{\tau_{\mathrm{p}}}+\frac{2}{7} \frac{\tau}{\tau_{\mathrm{p}}^{\prime}}\right) \\
a_{2} \simeq-\frac{1}{\tau}\left(1+\frac{\tau}{\tau_{\mathrm{r}}}+\frac{2}{7} \frac{\tau}{\tau_{\mathrm{p}}}+\frac{5}{7} \frac{\tau}{\tau_{\mathrm{p}}^{\prime}}\right) \\
a_{3}=0
\end{array}\right.
$$

(dans $a_{1}$ et $a_{2}$ on a négligé pour simplifier les termes d'ordre 2 ou plus en $\left.\tau / \tau_{\mathbf{r}}, \tau / \tau_{\mathbf{p}}, \tau / \tau_{\mathbf{p}}^{\prime}\right)$. Nous obtenons donc deux constantes de temps courtes, de l'ordre de $\tau$, et une constante de temps infinie. Bien sûr, cette valeur infinie n'a pas de sens physique, et provient de l'approximation à l'ordre le plus bas en $\tau / T$ et $\tau / T_{\mathrm{r}}$ que nous avons faite; dans le paragraphe 2.1.2 suivant, nous préciserons cette constante de temps en gardant les termes du premier ordre, et nous verrons qu'elle est beaucoup plus longue que $\tau$, mais reste finie.

Les constantes de temps correspondant à $a_{1}$ et $a_{2}$ sont associées à l'évolution des deux orientations dans le niveau métastable (ceci peut être vérifié à partir de l'expression (2.1) de la matrice $A$ ). Il faut noter que, $\left\langle F_{z}\right\rangle_{3 / 2}$ et $\left\langle F_{z}\right\rangle_{1 / 2}$ étant couplées, chacune de ces constantes de temps ne concerne pas l'une seulement de ces deux orientations, mais un
« mode propre» d'évolution où toutes deux participent. Pour la dernière valeur propre $a_{3}$, il n'est pas étonnant qu'elle soit nulle puisque à l'ordre zéro en $\tau / T, \tau / T_{\mathrm{r}}$ l'évolution de $\left\langle I_{z}\right\rangle_{\mathrm{f}}$ s'écrit simplement :

$$
\frac{\mathrm{d}}{\mathrm{d} t}\left\langle I_{z}\right\rangle_{\mathrm{f}}=0
$$

l'orientation du niveau fondamental étant donc, en première approximation, constante. Cependant, le mode propre associé à $a_{3}$ (mode lent) n'intéresse pas uniquement $\left\langle I_{z}\right\rangle_{\mathrm{f}}$, mais également les orientations $\left\langle F_{z}\right\rangle_{3 / 2}$ et $\left\langle F_{z}\right\rangle_{1 / 2}$; précisons donc les caractéristiques de ce mode.

2.1.2 Caractéristiques du mode lent. - Si l'on s'intéresse à l'évolution du système sur des grands intervalles de temps $(t \gg \tau)$, le mode propre de constante de temps longue joue un rôle prépondérant. 
Dans ce mode, on a

$$
\begin{aligned}
& \frac{\mathrm{d}}{\mathrm{d} t}\left\langle F_{z}\right\rangle_{3 / 2}=a_{3}\left\langle F_{z}\right\rangle_{3 / 2}, \\
& \frac{\mathrm{d}}{\mathrm{d} t}\left\langle F_{z}\right\rangle_{1 / 2}=a_{3}\left\langle F_{z}\right\rangle_{1 / 2},
\end{aligned}
$$

ce qui donne puisque $a_{3}=0$ :

$$
\begin{aligned}
-\left[\frac{4}{9 \tau}+\frac{1}{\tau_{\mathrm{r}}}+\frac{1}{\tau_{\mathrm{p}}}\right]\left\langle F_{z}\right\rangle_{3 / 2}+\frac{10}{9 \tau}\left\langle F_{z}\right\rangle_{1 / 2}+ \\
+\frac{10}{9 \tau}\left\langle I_{z}\right\rangle_{\mathrm{f}}+\frac{\Phi}{\tau_{\mathrm{p}}}=0 \\
\frac{1}{9 \tau}\left\langle F_{z}\right\rangle_{3 / 2}-\left[\frac{7}{9 \tau}+\frac{1}{\tau_{\mathrm{r}}}+\frac{1}{\tau_{\mathrm{p}}}\right]\left\langle F_{z}\right\rangle_{1 / 2}- \\
-\frac{1}{9 \tau}\left\langle I_{z}\right\rangle_{\mathrm{f}}+\frac{\Phi^{\prime}}{\tau_{\mathrm{p}}^{\prime}}=0
\end{aligned}
$$

๙) Commençons par négliger dans ces équations les termes en $1 / \tau_{\mathrm{p}}, 1 / \tau_{\mathrm{p}}^{\prime}$ et $1 / \tau_{\mathrm{r}}$, qui sont très petits devant ceux en $1 / \tau$. Les valeurs moyennes $\left\langle F_{z}\right\rangle_{3 / 2}$, $\left\langle F_{z}\right\rangle_{1 / 2}$ et $\left\langle I_{z}\right\rangle_{\mathrm{f}}$ sont alors proportionnelles, et peuvent s'exprimer en fonction de l'une d'entre elles, disons $\left\langle I_{z}\right\rangle_{\mathrm{f}}$ :

$$
\left\{\begin{array}{l}
\left\langle F_{z}\right\rangle_{3 / 2}=\frac{10}{3}\left\langle I_{z}\right\rangle_{\mathrm{f}} \\
\left\langle F_{z}\right\rangle_{1 / 2}=\frac{1}{3}\left\langle I_{z}\right\rangle_{\mathrm{f}}
\end{array}\right.
$$

A une orientation nucléaire $\left\langle I_{z}\right\rangle_{\mathrm{f}}$ dans le niveau fondamental correspond donc une orientation dans le niveau $F=\frac{3}{2}$ nettement plus grande que dans le niveau $F=\frac{1}{2}$. On peut d'ailleurs s'interroger sur le signe de $\left\langle F_{z}\right\rangle_{1 / 2}$ puisque, comme nous l'avons vu plus haut, l'échange de métastabilité tend à transformer une orientation nucléaire du fondamental en une orientation opposée dans le niveau $F=\frac{1}{2}$ (terme en $-\frac{1}{9 \tau}\left\langle I_{z}\right\rangle_{\mathrm{f}}$ dans l'éq. (1.37b)). Cependant, il ne faut pas oublier qu'il existe un couplage fort entre les orientations des deux niveaux hyperfins sous l'effet de l'échange de métastabilité ; le niveau $F=\frac{1}{2}$ reçoit de cette façon une orientation appréciable du niveau $F=\frac{3}{2}$, qui est du même signe que $\left\langle I_{z}\right\rangle_{\mathrm{f}}$; cette orientation est plus grande que celle qui vient directement du fondamental, de sorte que $\left\langle F_{z}\right\rangle_{1 / 2}$ a même signe que $\left\langle I_{z}\right\rangle_{\mathrm{f}}$.

Remarquons que les égalités (2.5), reportées dans (1.9), indiquent que :

$$
\left\{\begin{array}{l}
\left\langle S_{z}\right\rangle_{\mathrm{m}}=\frac{8}{3}\left\langle I_{z}\right\rangle_{\mathrm{f}} \\
\left\langle I_{z}\right\rangle_{\mathrm{m}}=\left\langle I_{z}\right\rangle_{\mathrm{f}}
\end{array}\right.
$$

L'égalité entre $\left\langle I_{z}\right\rangle_{\mathrm{m}}$ et $\left\langle I_{z}\right\rangle_{\mathrm{f}}$ était prévisible : les collisions d'échange de métastabilité font passer dans le niveau métastable l'orientation nucléaire du fondamental; tant que l'égalité entre $\left\langle I_{z}\right\rangle_{\mathrm{m}}$ et $\left\langle I_{z}\right\rangle_{\mathrm{f}}$ n'est pas réalisée, l'orientation totale

$$
\langle\mathbf{F}\rangle=\langle\mathbf{S}\rangle_{\mathrm{m}}+\langle\mathbf{I}\rangle_{\mathrm{f}}
$$

de l'atome métastable après collision est différente de l'orientation $\langle\mathbf{S}\rangle_{\mathrm{m}}+\langle\mathbf{I}\rangle_{\mathrm{m}}$ avant collision et, comme le couplage hyperfin ne modifie pas $\langle\mathbf{F}\rangle$, les diverses orientations changent nécessairement sous l'effet global de la collision. L'équilibre ne peut donc être réalisé que si $\left\langle I_{z}\right\rangle_{\mathrm{f}}=\left\langle I_{z}\right\rangle_{\mathrm{m}}$.

$\beta)$ Si l'on garde dans (2.4) les termes en $1 / \tau_{\mathrm{p}}$, $1 / \tau_{\mathrm{p}}^{\prime}, 1 / \tau_{\mathbf{r}}$, on obtient :

avec :

$$
\left\{\begin{array}{l}
\left\langle F_{z}\right\rangle_{3 / 2}=\frac{1}{D}\left\{5\left(\frac{2}{3}+\frac{\tau}{\tau_{\mathrm{r}}}+\frac{\tau}{\tau_{\mathrm{p}}^{\prime}}\right)\left\langle I_{z}\right\rangle_{\mathrm{f}}+\frac{1}{2}\left(7+9 \frac{\tau}{\tau_{\mathrm{r}}}+9 \frac{\tau}{\tau_{\mathrm{p}}^{\prime}}\right) \frac{\tau}{\tau_{\mathrm{p}}} \Phi+5 \frac{\tau}{\tau_{\mathrm{p}}^{\prime}} \Phi^{\prime}\right\} \\
\left\langle F_{z}\right\rangle_{1 / 2}=\frac{1}{D}\left\{\frac{1}{2}\left(\frac{2}{3}-\frac{\tau}{\tau_{\mathrm{r}}}-\frac{\tau}{\tau_{\mathrm{p}}}\right)\left\langle I_{z}\right\rangle_{\mathrm{f}}+\frac{1}{2} \frac{\tau}{\tau_{\mathrm{p}}} \Phi+\frac{1}{2}\left(4+9 \frac{\tau}{\tau_{\mathrm{r}}}+9 \frac{\tau}{\tau_{\mathrm{p}}}\right) \frac{\tau}{\tau_{\mathrm{p}}^{\prime}} \Phi^{\prime}\right\}
\end{array}\right.
$$

$$
D=1+\frac{7}{2}\left(\frac{\tau}{\tau_{\mathrm{r}}}+\frac{\tau}{\tau_{\mathrm{p}}}\right)+2\left(\frac{\tau}{\tau_{\mathrm{r}}}+\frac{\tau}{\tau_{\mathrm{p}}^{\prime}}\right)+\frac{9}{2}\left(\frac{\tau}{\tau_{\mathrm{r}}}+\frac{\tau}{\tau_{\mathrm{p}}}\right)\left(\frac{\tau}{\tau_{\mathrm{r}}}+\frac{\tau}{\tau_{\mathrm{p}}^{\prime}}\right) \text {. }
$$

Au premier ordre en $\tau / \tau_{\mathbf{r}}, \tau / \tau_{\mathrm{p}}, \tau / \tau_{\mathrm{p}}^{\prime}$, on obtient le résultat plus simple :

$$
\left\{\begin{array}{l}
\left\langle F_{z}\right\rangle_{3 / 2}=\left[\frac{10}{3}-\frac{35}{3}\left(\frac{\tau}{\tau_{\mathrm{p}}}+\frac{\tau}{\tau_{\mathrm{r}}}\right)-\frac{5}{3}\left(\frac{\tau}{\tau_{\mathrm{p}}^{\prime}}+\frac{\tau}{\tau_{\mathrm{r}}}\right)\right]\left\langle I_{z}\right\rangle_{\mathrm{f}}+\frac{7}{2} \frac{\tau}{\tau_{\mathrm{p}}} \Phi+5 \frac{\tau}{\tau_{\mathrm{p}}^{\prime}} \Phi^{\prime} \\
\left\langle F_{z}\right\rangle_{1 / 2}=\left[\frac{1}{3}-\frac{5}{3}\left(\frac{\tau}{\tau_{\mathrm{p}}}+\frac{\tau}{\tau_{\mathrm{r}}}\right)-\frac{2}{3}\left(\frac{\tau}{\tau_{\mathrm{p}}^{\prime}}+\frac{\tau}{\tau_{\mathrm{r}}}\right)\right]\left\langle I_{z}\right\rangle_{\mathrm{f}}+\frac{1}{2} \frac{\tau}{\tau_{\mathrm{p}}} \Phi+2 \frac{\tau}{\tau_{\mathrm{p}}^{\prime}} \Phi^{\prime} .
\end{array}\right.
$$

Ces équations nous montrent comment les processus de relaxation et de pompage dans le niveau métastable modifient les valeurs $(2.5)$ : la relaxation diminue le coefficient de $\left\langle I_{z}\right\rangle_{\mathrm{f}}$, les termes de pompage en $\Phi$ et $\Phi^{\prime}$ déplacent l'équilibre dans un sens qui dépend du signe de $\Phi$ et $\Phi^{\prime}$. 
2.2 CALCUl AU PREMIER ORDRE; TEMPS DE CONSTRUCTION DE L'ORIENTATION NUCLÉAIRE. - A l'ordre 1 en $\tau / T, \tau / T_{\mathrm{r}}$, les valeurs propres $a_{1}$ et $a_{2}$ sont modifiées de quantités négligeables en valeur relative; nous conserverons donc les valeurs données par (2.2a) et $(2.2 b)$. Par contre, la valeur propre $a_{3}$, qui est nulle si on se limite à l'ordre 0 , reçoit une contribution essentielle des termes du premier ordre en $\tau / T, \tau / T_{\mathrm{r}}$, et nous ne pouvons garder l'égalité $(2.2 c)$. De façon générale, toutes les quantités obtenues au paragraphe 1 précédent et qui ne sont pas nulles seront très peu modifiées par les termes du premier ordre, et il est inutile de reprendre le calcul; par exemple, nous conserverons ici les éq. (2.7) qui donnent les caractéristiques du mode lent. Nous n'allons donc reprendre que les équations qui nous ont donné zéro au paragraphe précédent, et chercher en particulier ce que deviennent au premier ordre les éq. (2.2c) et (2.3).

Pour cela, nous allons comme plus haut nous intéresser au mode lent, en supposant que le système est étudié sur des temps longs $(t \gg \tau)$, cas auquel les éq. (2.7) sont valables. Bien sûr, ces équations ne tiennent compte que des termes d'ordre zéro mais, si on les reporte dans (1.47a) qui donne $\mathrm{d}\left\langle I_{z}\right\rangle_{\mathrm{f}} / \mathrm{d} t$ (et dont les coefficients sont du premier ordre), on obtient une égalité valable au premier ordre :

$$
\begin{aligned}
\frac{\mathrm{d}}{\mathrm{d} t}\left\langle I_{z}\right\rangle_{\mathrm{f}}=-\left[\frac{1}{T}+\frac{1}{T_{\mathrm{r}}}\right]\left\langle I_{z}\right\rangle_{\mathrm{f}}+ & \frac{1}{3 D T}\left\{\left[3+\frac{11}{2} \frac{\tau}{\tau_{\mathrm{r}}}+\frac{1}{2} \frac{\tau}{\tau_{\mathrm{p}}}+5 \frac{\tau}{\tau_{\mathrm{p}}^{\prime}}\right]\left\langle I_{z}\right\rangle_{\mathrm{f}}+\right. \\
+ & \left.\frac{1}{2}\left[6+9 \frac{\tau}{\tau_{\mathrm{r}}}+9 \frac{\tau}{\tau_{\mathrm{p}}^{\prime}}\right] \frac{\tau}{\tau_{\mathrm{p}}} \Phi+\frac{1}{2}\left[6-9 \frac{\tau}{\tau_{\mathrm{r}}}-9 \frac{\tau}{\tau_{\mathrm{p}}}\right] \frac{\tau}{\tau_{\mathrm{p}}^{\prime}} \Phi^{\prime}\right\},
\end{aligned}
$$

c'est-à-dire :

$$
\frac{\mathrm{d}}{\mathrm{d} t}\left\langle I_{z}\right\rangle_{\mathrm{f}}=\frac{1}{T_{1}}\left[I_{0}-\left\langle I_{z}\right\rangle_{\mathrm{f}}\right]
$$

avec :

$$
\begin{aligned}
\frac{1}{T_{1}} & =\frac{1}{T_{\mathrm{r}}}+\frac{1}{T}\left\{1-\frac{1}{D}\left(1+\frac{11}{6} \frac{\tau}{\tau_{\mathrm{r}}}+\frac{1}{6} \frac{\tau}{\tau_{\mathrm{p}}}+\frac{5}{3} \frac{\tau}{\tau_{\mathrm{p}}^{\prime}}\right)\right\} \\
& =\frac{1}{T_{\mathrm{r}}}+\frac{1}{T}\left\{\frac{11}{3 D} \frac{\tau}{\tau_{\mathrm{r}}}+\frac{10}{3 D} \frac{\tau}{\tau_{\mathrm{p}}}+\frac{1}{3 D} \frac{\tau}{\tau_{\mathrm{p}}^{\prime}}+\frac{9}{2 D}\left(\frac{\tau}{\tau_{\mathrm{r}}}+\frac{\tau}{\tau_{\mathrm{p}}}\right)\left(\frac{\tau}{\tau_{\mathrm{r}}}+\frac{\tau}{\tau_{\mathrm{p}}^{\prime}}\right)\right\},
\end{aligned}
$$

et :

$$
I_{0}=\frac{T_{1}}{D T}\left\{\left[1+\frac{3}{2} \frac{\tau}{\tau_{\mathrm{r}}}+\frac{3}{2} \frac{\tau}{\tau_{\mathrm{p}}^{\prime}}\right] \frac{\tau}{\tau_{\mathrm{p}}} \Phi+\left[1-\frac{3}{2} \frac{\tau}{\tau_{\mathrm{r}}}-\frac{3}{2} \frac{\tau}{\tau_{\mathrm{p}}}\right] \frac{\tau}{\tau_{\mathrm{p}}^{\prime}} \Phi^{\prime}\right\} .
$$

La valeur propre $a_{3}$ est donc, au premier ordre, égale à la quantité $1 / T_{1}$ donnée par (2.12); $T_{1}$ est le temps de construction de l'orientation nucléaire dans l'état fondamental. Lorsque $t \gg T_{1}$, on a $\left\langle I_{z}\right\rangle_{\mathrm{f}}=I_{0}$; l'égalité (2.13) donne donc l'orientation nucléaire limite obtenue par pompage optique.

Comme $\tau / \tau_{\mathbf{r}}, \tau / \tau_{\mathfrak{p}}, \tau / \tau_{\mathfrak{p}}^{\prime} \ll 1$, on peut souvent remplacer (2.12) et (2.13) par leurs développements à l'ordre le plus bas par rapport à ces quantités; il vient alors :

$$
I_{0}=\frac{T_{1}}{T}\left(\frac{\tau}{\tau_{\mathrm{p}}} \Phi+\frac{\tau}{\tau_{\mathrm{p}}^{\prime}} \Phi^{\prime}\right)=\frac{n}{N}\left(\frac{T_{1}}{\tau_{\mathrm{p}}} \Phi+\frac{T_{1}}{\tau_{\mathrm{p}}} \Phi^{\prime}\right) \text {. }
$$

La formule (2.14) est semblable, à des coefficients près, à celle que donne la théorie phénoménologique de Schearer et al. (réf. [20], formule (10)); le terme en $\frac{11}{3 T} \frac{\tau}{\tau_{\mathrm{r}}}$ apparaît d'ailleurs dans l'étude du pompage optique de Fitzsimmons (réf. [22], pages 17 et 18). On peut remarquer sur (2.14) que, si le niveau métastable ne subit aucune relaxation $\left(\tau / \tau_{\mathrm{r}}=\tau / \tau_{\mathrm{p}}=\tau / \tau_{\mathrm{p}}^{\prime}=0\right)$, le temps $T_{1}$ est simplement égal à̀ $T_{\mathrm{r}}$. L'échange de métastabilité ne produit donc par lui-même aucune évolution (relaxation ou pompage) du niveau fondamental. D'un point de vue physique, ceci se comprend bien puisque, s'il était seul, l'échange de métastabilité égaliserait dès que $t \gg \tau$ l'orientation nucléaire du niveau métastable à celle du fondamental, ne pouvant ainsi plus faire évoluer ultérieurement cette dernière. Ce sont donc les processus de relaxation et de pompage qui, en déplaçant comme le montrent (2.7) et (2.9) les valeurs d'équilibre de $\left\langle F_{z}\right\rangle_{3 / 2}$ et $\left\langle F_{z}\right\rangle_{1 / 2}$, sont responsables d'une évolution supplémentaire de $\left\langle I_{z}\right\rangle_{\mathrm{f}}$; les trois derniers termes de (2.14) montrent comment l'échange "ramène " dans le niveau fondamental la relaxation du niveau métastable, avec des coefficients de l'ordre de $\tau / T=n / N=10^{-6}$; ces coefficients sont très petits, et c'est pourquoi on 
trouve des temps $T_{1}$ très longs, allant de 10 secondes à plusieurs minutes.

Bien sûr, à la limite où $\tau_{\mathrm{r}} \rightarrow 0$, on trouve en utilisant (2.8) et (2.12) que $\frac{1}{T_{1}}$ est simplement égal à $\frac{1}{T_{\mathrm{r}}}+\frac{1}{T}$ : si le niveau métastable est totalement désorienté, chaque échange de métastabilité se traduit par une relaxation pour le fondamental.

3. Orientations transversales ; effets liés à la circulation de cohérence. - Comme l'évolution des orientations longitudinales, l'évolution des orientations transversales est profondément affectée par l'échange de métastabilité. Or il est important de connaître le comportement de ces orientations transversales, par exemple dans les expériences où l'on détecte les variations transitoires de $\left\langle F_{x}\right\rangle_{3 / 2}$ à la suite d'une impulsion de radiofréquence. De plus, comme nous le verrons au paragraphe 4 , les fréquences propres des modes associés aux orientations transversales donnent les positions des raies de résonance magnétique extrapolées à intensité de radiofréquence nulle. Nous allons donc dans ce paragraphe étudier l'évolution des orientations transversales. Celle-ci est déterminée par l'éq. (1.46b); nous procéderons en deux temps comme dans le paragraphe précédent.

3.1 CAlCUl A L'ORDRE zÉRo. - A l'ordre zéro en $\tau / T, \tau / T_{\mathrm{r}}$, la matrice $B$ s'écrit :

$[(B)]=\frac{1}{\tau}\left[\begin{array}{cccc}-\frac{4}{9}+\frac{2 i \omega_{\mathrm{m}} \tau}{3} & \frac{10}{9} & \frac{10}{9} \\ \frac{1}{9} & -\frac{7}{9}+\frac{4 i \omega_{\mathrm{m}} \tau}{3} & -\frac{1}{9} \\ 0 & 0 & i \omega_{\mathrm{f}} \tau\end{array}\right]$

(nous avons négligé les termes en $\tau / \tau_{\mathbf{r}}, \tau / \tau_{\mathbf{p}}$ et $\tau / \tau_{\mathbf{p}}^{\prime}$ dans un but de simplification; il n'y aurait cependant aucune difficulté de principe à les garder). Les trois valeurs propres de cette matrice sont :

$\left\{\begin{array}{l}b_{1}=\frac{1}{\tau}\left\{-\frac{11}{18}+i \omega_{\mathrm{m}} \tau+\alpha\left(\omega_{\mathrm{m}} \tau\right)+i \beta\left(\omega_{\mathrm{m}} \tau\right)\right\} \\ b_{2}=\frac{1}{\tau}\left\{-\frac{11}{18}+i \omega_{\mathrm{m}} \tau-\alpha\left(\omega_{\mathrm{m}} \tau\right)-i \beta\left(\omega_{\mathrm{m}} \tau\right)\right\} \\ b_{3}=i \omega_{\mathrm{f}} .\end{array}\right.$

Les nombres réels $\alpha$ et $\beta$ étant définis par

$$
\begin{aligned}
& {\left[\alpha\left(\omega_{\mathrm{m}} \tau\right)+i \beta\left(\omega_{\mathrm{m}} \tau\right)\right]^{2}=} \\
& \quad=\left(\frac{7}{18}\right)^{2}-\frac{i \omega_{\mathrm{m}} \tau}{9}\left(1-i \omega_{\mathrm{m}} \tau\right)
\end{aligned}
$$

(on suppose $\alpha>0$ pour achever de déterminer $\alpha$ et $\beta$ ). Cette fois, les valeurs propres de la matrice sont complexes : chacun des modes propres des trois orientations transversales couplées est amorti et possède également une fréquence propre d'oscillation donnée (à un facteur $2 \pi$ près) par les parties imaginaires de $b_{1}, b_{2}$ et $b_{3}$. Comme pour les orientations longitudinales, les deux premières valeurs propres sont uniquement associées aux évolutions des orientations dans le niveau métastable, la valeur propre $b_{3}$ étant associée à un mode qui concerne à la fois les trois orientations $\left\langle F_{+}\right\rangle_{3 / 2},\left\langle F_{+}\right\rangle_{1 / 2}$ et $\left\langle I_{+}\right\rangle_{\mathrm{f}}$.

3.1 .1 Les constantes de temps et fréquences propres d'évolution. - Nous allons principalement étudier ici les variations de $b_{1}$ et $b_{2}$, la valeur propre $b_{3}$ valant simplement $i \omega_{\mathrm{f}}$ (dans l'approximation à l'ordre zéro). Les deux modes associés à $b_{1}$ et $b_{2}$ ont des constantes de temps d'amortissement courtes, de l'ordre de $\tau$, et des fréquences propres de l'ordre de $\omega_{\mathrm{m}}$. Le calcul de ces constantes de temps et de ces fréquences propres est aisé à partir de (3.2) et (3.3). Commençons tout d'abord par un cas limite simple, fréquemment réalisé en pratique, celui où le champ magnétique statique est assez intense pour que (') $\gg 1 / \tau$.

๔) Valeurs en champ fort. - Lorsque $\omega_{\mathrm{m}} \tau \gg 1$, on peut développer à partir de la définition (3.3) $\alpha$ et $\beta$ en puissances de $1 / \omega_{\mathrm{m}} \tau$; en reportant ce résultat dans $b_{1}$ et $b_{2}$, on obtient :

$$
\left\{\begin{aligned}
& b_{1}=\frac{1}{\tau}\left\{\left[-\frac{4}{9}+\frac{5}{54\left(\omega_{\mathrm{m}} \tau\right)^{2}}+\cdots\right]+\right. \\
&\left.+i\left[\frac{2 \omega_{\mathrm{m}} \tau}{3}+\frac{5}{27 \omega_{\mathrm{m}} \tau}+\cdots\right]\right\}, \\
& b_{2}=\frac{1}{\tau}\left\{\left[-\frac{7}{9}-\frac{5}{54\left(\omega_{\mathrm{m}} \tau\right)^{2}}+\cdots\right]+\right. \\
&\left.+i\left[\frac{4 \omega_{\mathrm{m}} \tau}{3}-\frac{5}{27 \omega_{\mathrm{m}} \tau}+\cdots\right]\right\} .
\end{aligned}\right.
$$

En l'absence d'échange de métastabilité $(\tau=\infty)$, on aurait $b_{1}=2 i \omega_{\mathrm{m}} / 3$ et $b_{2}=4 i \omega_{\mathrm{m}} / 3$; les deux modes propres correspondent simplement aux orientations $\left\langle F_{+}\right\rangle_{3 / 2}$ et $\left\langle F_{+}\right\rangle_{1 / 2}$, et ont pour fréquence propre les fréquences de résonance dans les deux sous-niveaux $F=\frac{3}{2}$ et $F=\frac{1}{2}$. Les formules (3.4) montrent comment l'échange de métastabilité modifie ces fréquences propres, et fait apparaître un amortissement. En fait, tant que l'on peut négliger les termes en $1 / \omega_{\mathrm{m}} \tau$, le seul effet de l'échange est de donner une durée de vie $9 \tau / 4$ à $\left\langle F_{+}\right\rangle_{3 / 2}$ (premier mode), $9 \tau / 7$ à $\left\langle F_{+}\right\rangle_{1 / 2}$ (second mode); ceci provient de ce que l'orientation transversale qui part de chaque niveau $F$ ne peut y revenir (elle précesse autour de $\mathbf{B}_{0}$ dans l'autre sous-niveau hyperfin avec une fréquence complètement différente, de sorte qu'elle revient dans le niveau initial avec une phase aléatoire répartie uniformément entre 0 et $2 \pi$; en termes de matrice densité, on dit qu'il n'y a pas « circulation de cohérence »). Il n'est donc pas étonnant que l'on retrouve dans ce cas les facteurs $\frac{4}{9}$ et $\frac{7}{9}$, correspondant d'après (1.40) à la proportion de l'orientation qui est détruite dans chaque sous-niveau $F$ par une collision d'échange 
de métastabilité; le passage de l'orientation dans l'autre sous-niveau donne un terme de couplage « non séculaire " qui ne joue aucun rôle.

Lorsque les termes en $1 / \omega_{\mathrm{m}} \tau$ ne sont plus négligeables, les modes propres sont modifiés (chacun d'entre eux correspond à une combinaison linéaire de $\left\langle F_{+}\right\rangle_{3 / 2}$ et $\left\langle F_{+}\right\rangle_{1 / 2}$ ), ainsi que leurs valeurs propres. Les formules (3.4) montrent comment sont affectées, aux ordres les plus bas en $1 / \omega_{\mathrm{m}} \tau$, les fréquences propres et les amortissements; on constate notamment que les fréquences propres se rapprochent. Ce dernier effet est lié à une faible "circulation de cohérence " entre les sous-niveaux $F=\frac{3}{2}$ et $F=\frac{1}{2}$. Rappelons que de tels effets sont classiques en pompage optique, la circulation de cohérence étant généralement assurée par l'irradiation lumineuse (déplacements des résonances liés aux transitions réelles [13]).

ß) Valeur en champ magnétique quelconque. - On a représenté sur les figures 3 et 4 l'allure des variations en fonction de $\omega_{\mathrm{m}}$ des parties réelles et imaginaires de $b_{1}$ et $b_{2}$. On voit que les amortissements des deux modes varient en sens opposé : l'un est fonction croissante du champ magnétique, l'autre décroissante. Quant aux fréquences propres des deux modes, leur variation n'est pas linéaire en fonction de $\omega_{\mathrm{m}}$, comme ce serait le cas en l'absence du couplage entre les orientations transversales; lorsque $\omega_{\mathrm{m}}$ diminue, les fréquences propres tendent à se rapprocher et, lorsque $\omega_{\mathrm{m}} \tau \lesssim 1$ (condition qui autorise une circulation de cohérence importante entre les deux sous-niveaux $F=\frac{3}{2}$ et $F=\frac{1}{2}$ ), elles sont très différentes des fré-

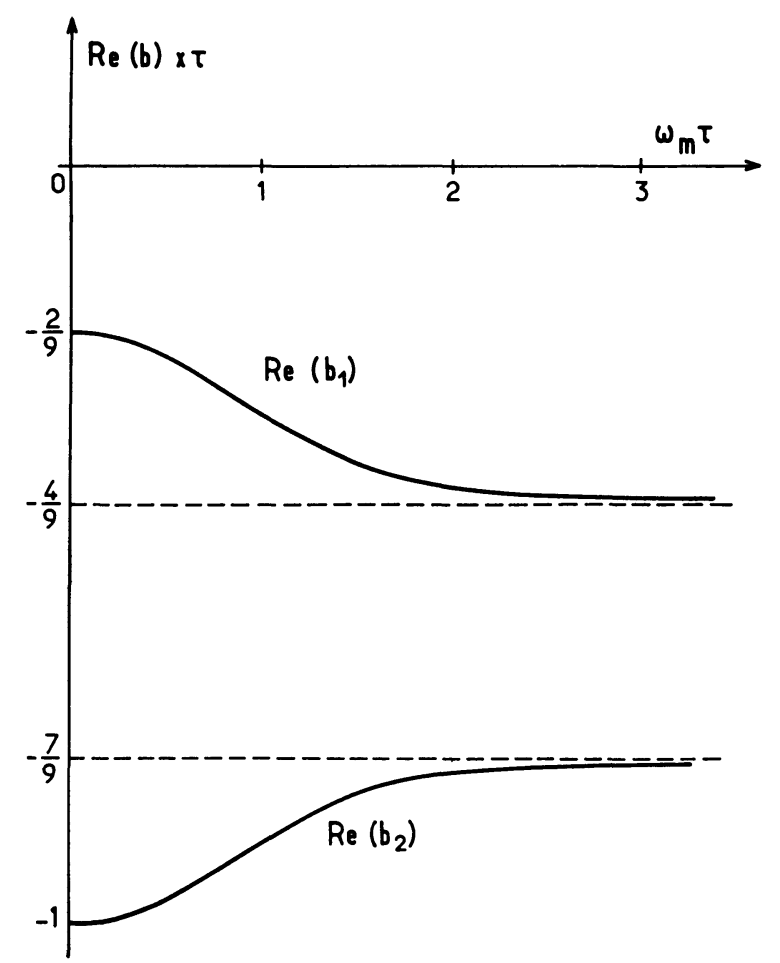

FIG. 3. - Variations en fonction du champ magnétique des taux de relaxation des deux modes associés aux orientations transversales du niveau métastable.

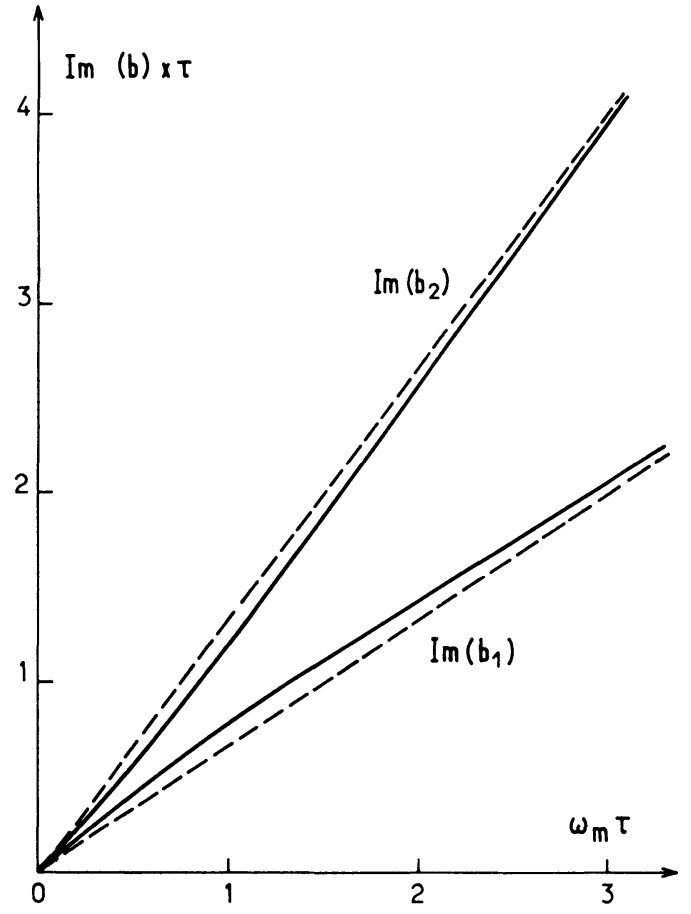

FIG. 4. - Variations en fonction du champ magnétique des fréquences propres des deux modes associés aux orientations transversales du niveau métastable.

quences Zeeman $\frac{2}{3} \frac{\omega_{\mathrm{m}}}{2 \pi}$ et $\frac{4}{3} \frac{\omega_{\mathrm{m}}}{2 \pi}$ des deux sousniveaux hyperfins. Dans le cas limite où $\omega_{\mathrm{m}} \tau \ll 1$, on calcule :

$$
\left\{\begin{array}{r}
b_{1}=\frac{1}{\tau}\left\{\left[-\frac{2}{9}-\frac{40}{343}\left(\omega_{\mathrm{m}} \tau\right)^{2}+\cdots\right]+\right. \\
\left.+\frac{6}{7} i \omega_{\mathrm{m}} \tau+\cdots\right\} \\
b_{2}=\frac{1}{\tau}\left\{\left[-1+\frac{40}{343}\left(\omega_{\mathrm{m}} \tau\right)^{2}+\cdots\right]+\right. \\
\left.+\frac{8}{7} i \omega_{\mathrm{m}} \tau+\cdots\right\}
\end{array} .\right.
$$

Pour $\omega_{\mathrm{m}}=0$, ces expressions donnent $b_{1}=-2 / 9 \tau$ et $b_{2}=-1 / \tau$; on retrouve les premiers termes de $(2.2 a)$ et $(2.2 b)$, ce qui est normal puisqu'en champ nul rien ne distingue les orientations longitudinales et transversales.

3.1.2 Caractéristiques du mode lent. - La valeur propre $b_{3}$ est associée à un mode non amorti, du moins à l'ordre zéro en $\tau / T, \tau / T_{\mathrm{r}}$ (nous calculerons son amortissement au premier ordre dans le paragraphe 3.2 suivant). Pour étudier les caractéristiques de ce mode, supposons, comme dans le paragraphe 2.1.2 précédent, que $t \gg \tau$, de sorte que le mode lent est seul à subsister. On a alors

$$
\frac{\mathrm{d}}{\mathrm{d} t}\left\langle F_{+}\right\rangle_{3 / 2}=i \omega_{\mathrm{f}}\left\langle F_{+}\right\rangle_{3 / 2},
$$


et des égalités semblables pour $\left\langle F_{+}\right\rangle_{1 / 2}$ et $\left\langle I_{+}\right\rangle_{\mathrm{f}}$; en reportant ces équations dans $(1.46 b)$, on obtient un système de 3 équations linéaires homogènes dont la solution s'écrit $\left({ }^{6}\right)$ :

$\left\{\begin{array}{l}\left\langle F_{+}\right\rangle_{3 / 2}=\frac{10}{9^{\prime}}\left\{\frac{1}{1-i \omega_{\mathrm{m}} \tau}+\frac{2}{1-4 i \omega_{\mathrm{m}} \tau}\right\}\left\langle I_{+}\right\rangle_{\mathrm{f}} \\ \left\langle F_{+}\right\rangle_{1 / 2}=\frac{1}{9}\left\{-\frac{2}{1-i \omega_{\mathrm{m}} \tau}+\frac{5}{1-4 i \omega_{\mathrm{m}} \tau}\right\}\left\langle I_{+}\right\rangle_{\mathrm{f}} .\end{array}\right.$

Ces égalités sont les analogues, pour les orientations transversales, des éq. (2.5). Cependant, les coefficients de (3.6) sont complexes, ce qui traduit l'existence d'un déphasage entre les orientations transversales des divers niveaux.

3.2 CAlCUl aU PREMiER ORdRE; DÉPlaCEMENT DE LA FRÉQUENCE DU NIVEAU FONDAMENTAL. - Au premier ordre en $\tau / T, \tau / T_{\mathrm{r}}$, les valeurs propres $b_{1}$ et $b_{2}$ sont modifiées de quantités négligeables. Par contre, $b_{3}$ peut subir des modifications importantes. Pour les calculer, procédons comme dans le paragraphe 2.2 pour les orientations longitudinales et reportons (3.6) dans la troisième ligne de $(1.46 b)$; il vient :

$$
\begin{gathered}
\frac{\mathrm{d}}{\mathrm{d} t}\left\langle I_{+}\right\rangle_{\mathrm{f}}=-\left\{\frac{1}{T_{\mathrm{r}}}+\frac{1}{T}-\frac{4}{9 T} \frac{1}{1-i \omega_{\mathrm{m}} \tau}-\right. \\
\left.-\frac{5}{9 T} \frac{1}{1-4 i \omega_{\mathrm{m}} \tau}-i \omega_{\mathrm{f}}\right\}\left\langle I_{+}\right\rangle_{\mathrm{f}} .
\end{gathered}
$$

Dans cette expression, la partie réelle du coefficient qui apparaît au second membre donne le temps de relaxation transversal $T_{2}$ associé au mode lent; on trouve :

$$
\begin{aligned}
\frac{1}{T_{2}}=\frac{1}{T_{\mathrm{r}}}+\frac{1}{T}\left[1-\frac{4}{9}\right. & \frac{1}{1+\left(\omega_{\mathrm{m}} \tau\right)^{2}}- \\
& \left.-\frac{5}{9} \frac{1}{1+16\left(\omega_{\mathrm{m}} \tau\right)^{2}}\right] .
\end{aligned}
$$

$\left({ }^{6}\right)$ Dans (3.6), nous avons négligé $\omega_{\mathrm{f}}$ devant $\omega_{\mathrm{m}}$, ce qui introduit une erreur de l'ordre de $10^{-3}$ en valeur relative (c'est-à-dire comparable à celle que nous avons faite en négligeant le rôle du magnétisme nucléaire dans les facteurs de Landé des sous-niveaux $F=\frac{3}{2}$ et $\left.F=\frac{1}{2}\right)$.
$\mathrm{Si}$ on fait $\omega_{\mathrm{m}}=0$, on trouve $1 / T_{2}=1 / T_{\mathrm{r}}$; mais, dès que $\omega_{\mathrm{m}} \tau$ n'est plus négligeable devant $1, T_{2}$ se raccourcit notablement et tend vers $\frac{1}{T_{\mathrm{r}}}+\frac{1}{T} \simeq \frac{1}{T}$. La partie imaginaire du coefficient donne la fréquence d'évolution de l'orientation transversale; elle diffère de $\omega_{\mathrm{f}}$ par la quantité $\delta \omega_{\mathrm{f}}$ :

$\delta \omega_{\mathrm{f}}=\frac{4}{9 T} \frac{\omega_{\mathrm{m}} \tau}{1+\left(\omega_{\mathrm{m}} \tau\right)^{2}}+\frac{5}{9 T} \frac{4 \omega_{\mathrm{m}} \tau}{1+16\left(\omega_{\mathrm{m}} \tau\right)^{2}}$.

L'interprétation physique de ce déplacement de fréquence et du raccourcissement de $T_{2}$ est bien connue [24], [25]. Comme pour les déplacements de fréquence discutés au paragraphe 3.1 précédent, elle est liée à la circulation de cohérence entre les niveaux : lorsque l'orientation du niveau fondamental passe dans le niveau métastable, elle subit une précession plus rapide $\left(\omega_{\mathrm{m}}\right.$ et $\omega_{\mathrm{f}}$ sont de même signe et $\left.\omega_{\mathrm{m}} \gg \omega_{\mathrm{f}}\right)$, et l'orientation qui retombe dans le niveau fondamental est déphasée et en avance. Ceci explique l'augmentation de la fréquence propre associée au niveau fondamental et, la circulation de cohérence étant moins aisée en champ fort, le fait que $T_{2}$ diminue lorsque $\omega_{\mathrm{m}}$ augmente. Cette fois encore, ces effets peuvent être rapprochés de ceux qui résultent de la circulation de cohérence entre le niveau fondamental et le niveau excité d'un atome soumis à une irradiation lumineuse résonnante [13].

Notons que, d'après (3.8) et (3.9), on peut avoir $\delta \omega_{\mathrm{f}}>1 / T_{2}$ si $\omega_{\mathrm{m}} \tau$ est suffisamment petit; si, pour les résonances dans les sous-niveaux $F=\frac{3}{2}$ et $F=\frac{1}{2}$, la circulation de cohérence entre le niveau fondamental et le niveau métastable se traduit par des déplacements complètement négligeables devant leur largeur, il en va tout autrement pour la résonance dans le niveau fondamental : cette dernière peut subir un déplacement comparable ou même supérieur à sa largeur.

Signalons que d'autres expressions de $T_{2}$ et de $\delta \omega_{\mathrm{f}}$, à notre avis inexactes, existent dans la littérature [24], [26]. On trouvera dans la référence [27] une discussion complète de ces expressions, ainsi que les vérifications expérimentales des formules (3.8) et (3.9).

\section{Bibliographie}

[1] Colegrove, F. D., Schearer, L. D. et Walters, G. K., Phys. Rev. 132 (1963) 2561.

[2] Buckingham, R. A. and Dalgarno, D., Proc. Roy. Soc. A 213 (1952) 327 et 546.

[3] Brigman, G. H., Brient, S. J., Matsen, F. A., J. Chem. Phys. 34 (1961) 958.

[4] Matsen, F. A. et ScotT, D. R., Quantum Theory of Atoms, Molecules and the Solid State (Academic Press) 1966.

Poshusta, R. D. et Matsen, F. A., Phys. Rev. 132 (1963) 307.

[5] Colegrove, F. D., Schearer, L. D. et Walters, G. K., Phys. Rev. A 135 (1964) 353.
[6] Fugol', I. Ya. et PaKhomov, P. L., JETP Lett. 3 (1966) 254 ; Sov. Phys. JETP 26 (1966) 526.

[7] Fitzsimmons, W. A., Lane, N. F. et Walters, G. K., Phys. Rev. 174 (1968) 193.

Fitzsimmons, W. A., Thèse, Rice University (1968).

[8] Kolker, H. J. et Michels, H. H., J. Chem. Phys. 50 (1969) 1762.

[9] Kodaira, M. et Watanabe, T., J. Phys. Soc. Japan 27 (1969) 1301.

[10] Paкhomov, P. L. et FUgol', I. Ya., Sov. Phys., Doklady 13 (1968) 317. 
[11] Evans, S. A. et Lane, N. F., Phys. Rev. 188 (1969) 268.

[12] Dupont-Roc, J., Leduc, M. et Lalö̈, F., Phys. Rev. Lett. 27 (1971) 467.

[13] Barrat, J. P. et Cohen-Tannoudi, C., J. Phys. Rad. 7 (1961) 329 et 443.

Cohen-Tannoudj, C., Ann. Phys. 7 (1961) 423 et 464.

[14] Greenhow, R. C., Phys. Rev. 136A (1964) 660.

[15] Timsit, R. S. et Daniels, J. M., Can. J. Phys. 49 (1971) 545

[16] Rosner, S. D. et Pipkin, F. M., Phys. Rev. 5A (1972) 1901.

[17] Daniels, J. M. et Timsit, R. S., Can. J. Phys. 49 (1971) 539.

[18] Pavlović, M. et LaloË, F., J. Physique 31 (1970) 173. Lalö̈, F., Ann. Phys. 6 (1971) 5.

[19] Partridge, R. B. et Series, G. W., Proc., Phys. Soc. 88 (1966) 983.

[20] Schearer, L. D., Thèse, Rice University (1966), Xerox University Microfilms Inc., Ann Arbor, Michigan, $\mathrm{n}^{\circ}$ 66-10, 376.
[21] Byerly, H. R., Master and Ph. D. Thesis, Rice University (1965 et 1967), Xerox University Microfilms, $\mathrm{n}^{\circ}$ 67-13, 056.

[22] Fitzsimmons, W. A., Master Thesis, Rice University (1966).

[23] Fitzsimmons, W. A. et Walters, G. K., Phys. Rev. Lett., 19 (1967) 943.

Fitzsimmons, W. A., TANKersley, L. L. et Walters, G. K., Phys. Rev. 179 (1969) 156.

[24] Donszelmann, A., Thèse, Amsterdam (1970); Physica 56 (1971) 138.

[25] Schearer, L. D., Colegrove, F. D., Walters, G. K., Rev. Sci. Instr. 35 (1964) 767.

[26] Dehmelt, H. G., Rev. Sci. Instr. 35 (1964) 768.

[27] Dupont-Roc, J., C. R. Hebd. Sëan. Acad. Sci. B 273 (1971) 45 et 283. Thèse, Paris (1972). 